\title{
Noether currents for Eulerian variational principles in non-barotropic magnetohydrodynamics and topological conservations laws
}

\author{
Asher Yahalom ${ }^{1,2, \dagger}$ and Hong Qin ${ }^{2}$ \\ ${ }^{1}$ Department of Electrical and Electronic Engineering, Ariel University, Kiryat Hamada POB 3, \\ Ariel 40700, Israel \\ ${ }^{2}$ PPPL, Princeton University, Princeton, NJ 08543, USA
}

(Received 26 May 2020; revised 23 September 2020; accepted 30 September 2020)

We derive a Noether current for the Eulerian variational principle of ideal non-barotropic magnetohydrodynamics (MHD). It was shown previously that ideal non-barotropic MHD is mathematically equivalent to a five function field theory with an induced geometrical structure in the case that field lines cover surfaces and this theory can be described using a variational principle. Here we use various symmetries of the flow to derive topological constants of motion through the derived Noether current and discuss their implication for non-barotropic MHD.

Key words: topological fluid dynamics, variational methods

\section{Introduction}

Variational principles for magnetohydrodynamics (MHD) were introduced by previous authors both in Lagrangian and Eulerian form. Vladimirov \& Moffatt (1995) in a series of papers have discussed an Eulerian variational principle for incompressible MHD. However, their variational principle contained three more functions in addition to the seven variables which appear in the standard equations of incompressible MHD, which are the magnetic field $\boldsymbol{B}$, the velocity field $\boldsymbol{v}$ and the pressure $P$. Yahalom \& Lynden-Bell (2008) obtained an Eulerian Lagrangian principle for barotropic MHD which depends on only six functions. The variational derivative of this Lagrangian produced all the equations needed to describe barotropic MHD without any additional constraints. Yahalom (2010) has shown that for the barotropic case four functions will suffice. Moreover, it was shown that the cuts of some of those functions (Yahalom 2013a) are topological local conserved quantities.

Previous work was concerned only with barotropic MHD. Variational principles of non-barotropic MHD can be found in the work of Bekenstein \& Oron (2000) in terms of 15 functions, and Kats (2003) in terms of 20 functions. Holm \& Kupershmidt $(1983 a, b)$

†Email address for correspondence: asya @ ariel.ac.il 
developed the non-canonical Poisson bracket for MHD by using the magnetic vector potential in which the gauge was chosen, so that it is comoving. The Hamiltonian they used was derived from a Lagrangian depending on 16 functions. Later Holm, Marsden \& Ratiu (1998) developed an Eulerian action principle for many different physical systems, including MHD, using the so-called Euler-Poincaré action principle, in which the transformation between Lagrangian fluid labels and the Eulerian particle position and time is thought of as an infinite-dimensional Lie group or Lie pseudo-group. The Euler-Lagrange equations for the action result in the Eulerian MHD momentum equation. However, in this approach the variations are constrained by the group and are not free. Thus they are denoted as Euler-Poincaré equations. Morrison (1982) has suggested a Hamiltonian approach but this also depends on eight canonical variables (see table 2, Morrison (1982)).

It was shown that this number can be somewhat reduced. In Yahalom $(2016 a, b)$ it was demonstrated that only five functions will suffice to describe non-barotropic MHD, and that the reduced Lagrangian has a distinct geometrical structure including an induced metric.

The theorem of Noether dictates that for every continuous symmetry group of an action, the system must possess a conservation law. For example time translation symmetry results in the conservation of energy, while spatial translation symmetry results in the conservation of linear momentum and rotation symmetry in the conservation of angular momentum, to list some well known examples. But sometimes the conservation law is discovered without reference to Noether's theorem by using the equations of the system. In that case one is tempted to inquire what the hidden symmetry associated with this conservation law is, and what is the simplest way to represent it.

The concept of metage as a label for fluid elements along a vortex line in ideal fluids was first introduced by Lynden-Bell \& Katz (1981). A translation group of this label was found to be connected to the conservation of Moffat's (Vladimirov \& Moffatt 1995) helicity by Yahalom (1995) using a Lagrangian variational principle. The concept of metage was later generalized by Yahalom \& Lynden-Bell (2008) for barotropic MHD, but now as a label for fluid elements along magnetic field lines which are comoving with the flow in the case of ideal MHD. Yahalom \& Lynden-Bell (2008) have also shown that the translation group of the magnetic metage is connected to Woltjer conservation of cross-helicity for barotropic MHD (Woltjer 1958a,b). Recently the concept of metage was generalized also for non-barotropic MHD in which magnetic field lines lie on entropy surfaces (Yahalom 2017a). This was later generalized by dropping the entropy condition on magnetic field lines (Yahalom 2017d). In those papers the metage translation symmetry group was used to generate a non-barotropic cross-helicity generalization using a Lagrangian variational principle.

Cross-helicity was first described by Woltjer $(1958 a, b)$ and is given by

$$
H_{C} \equiv \int \boldsymbol{B} \cdot \boldsymbol{v} \mathrm{d}^{3} x,
$$

in which the integral is taken over the entire flow domain. Here $H_{C}$ is conserved for barotropic or incompressible MHD and is given a topological interpretation in terms of the knottiness of magnetic and flow field lines.

Both conservation laws for the helicity in the fluid dynamics case and the barotropic MHD case were shown to originate from a relabeling symmetry through Noether's theorem (Yahalom 1995; Padhye \& Morrison 1996a,b; Yahalom \& Lynden-Bell 2008). Webb et al. (2014b) have generalized the idea of relabeling symmetry to non-barotropic 
MHD and derived their generalized cross-helicity conservation law by using Noether's theorem, but without using the simple representation which is connected with the metage variable. The conservation law deduction involves a divergence symmetry of the action. These conservation laws were written as Eulerian conservation laws of the form $D_{t}+\nabla \cdot F=0$ where $\mathrm{D}$ is the conserved density and $\mathrm{F}$ is the conserved flux. Webb, McKenzie \& Zank (2015) discuss the cross-helicity conservation law for non-barotropic MHD in a multisymplectic formulation of MHD. Webb et al. $(2014 a, b)$ emphasize that the generalized cross-helicity conservation law in MHD, and the generalized helicity conservation law in non-barotropic fluids, are non-local in the sense that they depend on the auxiliary non-local variable $\sigma$, which depends on the Lagrangian time integral of the temperature $T(x, t)$. Note that a potential vorticity conservation equation for non-barotropic MHD was derived by Webb \& Mace (2015) by using Noether's second theorem.

Recently the non-barotropic cross-helicity was generalized using additional label translation symmetry groups ( $\chi$ and $\eta$ translations) (Yahalom 2019b), this led to additional topological conservation laws, the $\chi$ and $\eta$ cross-helicities.

Previous analysis depended on Lagrangian variational principles and their Noether currents. Here we introduce a novel approach based on an Eulerian variational principle. We derive the Noether current of the Eulerian variational principle and show how this can be used to derive topological conservation laws using label symmetries.

The plan of this paper is as follows. First we introduce the standard notation and equations of non-barotropic MHD. Next we introduce the Eulerian variational principle suitable for the non-barotropic case. This is followed by a derivation of the Noether current, and finally we use the Noether current to obtain the generalized non-barotropic cross-helicities. Implications for non-barotropic MHD dynamics of the topological conservation laws are discussed.

\section{Standard formulation of ideal non-barotropic MHD}

The standard set of equations solved for non-barotropic MHD are

$$
\begin{aligned}
& \frac{\partial \boldsymbol{B}}{\partial t}=\nabla \times(\boldsymbol{v} \times \boldsymbol{B}), \\
& \nabla \cdot \boldsymbol{B}=0, \\
& \frac{\partial \rho}{\partial t}+\nabla \cdot(\rho \boldsymbol{v})=0, \\
& \rho \frac{\mathrm{d} \boldsymbol{v}}{\mathrm{d} t}=\rho\left(\frac{\partial \boldsymbol{v}}{\partial t}+(\boldsymbol{v} \cdot \nabla) \boldsymbol{v}\right)=-\nabla p(\rho, s)+\frac{(\nabla \times \boldsymbol{B}) \times \boldsymbol{B}}{4 \pi}, \\
& \frac{\mathrm{d} s}{\mathrm{~d} t}=0,
\end{aligned}
$$

where $\partial / \partial t$ is the temporal derivative, $\mathrm{d} / \mathrm{d} t$ is the temporal material derivative and $\nabla$ has its standard meaning in vector calculus. Here $\rho$ is the fluid density, $s$ is the specific entropy and $p(\rho, s)$ is the pressure which depends on the density and entropy (the non-barotropic case). The justification for those equations and the conditions under which they apply can be found in standard books on MHD (see for example Sturrock (1994)). The number of independent variables for which one needs to solve is eight $(\boldsymbol{v}, \boldsymbol{B}, \rho, s)$ and the number of 
(2.1), (2.3), (2.4), (2.5) is also eight. Note that (2.2) is a condition on the initial $\boldsymbol{B}$ field and is satisfied automatically for any other time due to (2.1). We will find it useful to introduce the following thermodynamic equations for later use:

$$
\left.\begin{array}{c}
\mathrm{d} \varepsilon=T \mathrm{~d} s-p \mathrm{~d} \frac{1}{\rho}=T \mathrm{~d} s+\frac{p}{\rho^{2}} \mathrm{~d} \rho \\
\frac{\partial \varepsilon}{\partial s}=T, \quad \frac{\partial \varepsilon}{\partial \rho}=\frac{p}{\rho^{2}} \\
w=\varepsilon+\frac{p}{\rho}=\varepsilon+\frac{\partial \varepsilon}{\partial \rho} \rho=\frac{\partial(\rho \varepsilon)}{\partial \rho} \\
\mathrm{d} w=\mathrm{d} \varepsilon+\mathrm{d}\left(\frac{p}{\rho}\right)=T \mathrm{~d} s+\frac{1}{\rho} \mathrm{d} p
\end{array}\right\}
$$

where $\varepsilon$ is the specific internal energy, $T$ is the temperature and $w$ is the specific enthalpy. A special case of equation of state is the polytropic equation of state (Binney \& Tremaine 1987),

$$
p=K \rho^{\gamma},
$$

where $K$ and $\gamma$ may depend on the specific entropy $s$. Hence

$$
\frac{\partial \varepsilon}{\partial \rho}=K \rho^{\gamma-2} \Rightarrow \varepsilon=\frac{K}{\gamma-1} \rho^{\gamma-1}=\frac{p}{\rho(\gamma-1)} \Rightarrow \rho \varepsilon=\frac{p}{\gamma-1},
$$

where the last identity is up to a function dependent on $s$.

\section{Variational principle of non-barotropic MHD}

In the following section we will generalize the approach of Yahalom \& Lynden-Bell (2008) for the non-barotropic case (Yahalom 2016a,b). Consider the action

$$
\left.\begin{array}{c}
A \equiv \int \mathcal{L} d^{3} x \mathrm{~d} t, \\
\mathcal{L} \equiv \mathcal{L}_{1}+\mathcal{L}_{2}, \\
\mathcal{L}_{1} \equiv \rho\left(\frac{1}{2} \boldsymbol{v}^{2}-\varepsilon(\rho, s)\right)+\frac{\boldsymbol{B}^{2}}{8 \pi}, \\
\mathcal{L}_{2} \equiv v\left[\frac{\partial \rho}{\partial t}+\nabla \cdot(\rho \boldsymbol{v})\right]-\rho \alpha \frac{\mathrm{d} \chi}{\mathrm{d} t}-\rho \beta \frac{\mathrm{d} \eta}{\mathrm{d} t}-\rho \sigma \frac{\mathrm{d} s}{\mathrm{~d} t}-\frac{B}{4 \pi} \cdot \nabla \chi \times \nabla \eta .
\end{array}\right\}
$$

In the specific case of a polytropic equation of state we have, according to (2.8), that

$$
\mathcal{L}_{1}=\frac{1}{2} \rho \boldsymbol{v}^{2}-\frac{p}{\gamma-1}+\frac{\boldsymbol{B}^{2}}{8 \pi} .
$$

Obviously $v, \alpha, \beta, \sigma$ are Lagrange multipliers which were inserted in such a way that the variational principle will yield the following equations:

$$
\left.\begin{array}{c}
\frac{\partial \rho}{\partial t}+\nabla \cdot(\rho \boldsymbol{v})=0, \\
=0, \quad \rho \frac{\mathrm{d} \eta}{\mathrm{d} t}=0, \quad \rho \frac{\mathrm{d} s}{\mathrm{~d} t}=0 .
\end{array}\right\}
$$


It is not assumed that $\nu, \alpha, \beta, \sigma$ are single valued. Provided $\rho$ is not null those are just the continuity equation (2.3), entropy conservation and the conditions that Sakurai's functions are comoving. Taking the variational derivative with respect to $B$ we see that

$$
\boldsymbol{B}=\hat{\boldsymbol{B}} \equiv \nabla \chi \times \nabla \eta .
$$

Hence $B$ is in Sakurai's form (Sakurai 1979) and satisfies (2.2). It can be easily shown that provided that $\boldsymbol{B}$ is in the form given in (3.4) and (3.3) are satisfied, then also (2.1) is satisfied. We notice that the specific form of the magnetic field given in (3.4) appears under different names in the literature. The functions $\chi$ and $\eta$ are sometimes denoted 'Euler potentials', 'Clebsch variables' and also 'flux representation functions' (Hazeltine \& Meiss 2003). Equation (3.4) implies that the magnetic field lines lie on surfaces, the lines may be surface filling but not volume filling.

For the time being we have showed that all the equations of non-barotropic MHD can be obtained from the above variational principle except Euler's equations. We will now show that Euler's equations can be derived from the above variational principle as well. Let us take an arbitrary variational derivative of the above action with respect to $\boldsymbol{v}$, this will result in

$$
\begin{aligned}
\delta_{v} A= & \int \mathrm{d} t\left\{\int \mathrm{d}^{3} x \mathrm{~d} t \rho \delta \boldsymbol{v} \cdot[\boldsymbol{v}-\nabla v-\alpha \nabla \chi-\beta \nabla \eta-\sigma \nabla s]\right. \\
& \left.+\oint \mathrm{d} \boldsymbol{S} \cdot \delta \boldsymbol{v} \rho v+\int \mathrm{d} \boldsymbol{\Sigma} \cdot \delta \boldsymbol{v} \rho[v]\right\} .
\end{aligned}
$$

The integral $\oint \mathrm{d} \boldsymbol{S} \cdot \delta \boldsymbol{v} \rho v$ vanishes in many physical scenarios. In the case of astrophysical flows this integral will vanish since $\rho=0$ on the flow boundary, in the case of a fluid contained in a vessel no-flux boundary conditions $\delta \boldsymbol{v} \cdot \hat{n}=0$ are induced $(\hat{n}$ is a unit vector normal to the boundary). The surface integral $\int d \boldsymbol{\Sigma}$ on the cut of $v$ vanishes in the case that $v$ is single valued and $[v]=0$. In the case that $v$ is not single valued only a Kutta-type velocity perturbation (Yahalom, Pinhasi \& Kopylenko 2005) in which the velocity perturbation is parallel to the cut will cause the cut integral to vanish.

Provided that the surface integrals do vanish, and that $\delta_{v} A=0$ for an arbitrary velocity perturbation, we see that $v$ must have the following form:

$$
\boldsymbol{v}=\hat{\boldsymbol{v}} \equiv \nabla v+\alpha \nabla \chi+\beta \nabla \eta+\sigma \nabla s .
$$

The above equation is reminiscent of Clebsch representation in non-magnetic fluids. A similar expression was obtained by Morrison (Morrison 1982) using a Hamiltonian formalism, but in which the $s$ terms is replaced by $\psi$ which is conjugate to $s$. Vladimirov \& Moffatt (1995) have connected this type of representation and the Weber transformations in their $\S 3$; in fact their (3.16) resembles (3.6).

Let us now take the variational derivative with respect to the density $\rho$, we obtain

$$
\begin{aligned}
\delta_{\rho} A= & \int d^{3} x \mathrm{~d} t \delta \rho\left[\frac{1}{2} \boldsymbol{v}^{2}-w-\frac{\partial v}{\partial t}-\boldsymbol{v} \cdot \nabla v\right] \\
& +\int \mathrm{d} t \oint \mathrm{d} \boldsymbol{S} \cdot \boldsymbol{v} \delta \rho v+\int \mathrm{d} t \int \mathrm{d} \boldsymbol{\Sigma} \cdot \boldsymbol{v} \delta \rho[v]+\left.\int \mathrm{d}^{3} x v \delta \rho\right|_{t_{0}} ^{t_{1}},
\end{aligned}
$$

in which $w=\partial(\varepsilon \rho) / \partial \rho$ is the specific enthalpy. Hence, provided that $\oint \mathrm{d} \boldsymbol{S} \cdot \boldsymbol{v} \delta \rho v$ vanishes on the boundary of the domain and $\int \mathrm{d} \boldsymbol{\Sigma} \cdot \boldsymbol{v} \delta \rho[v]$ vanishes on the cut of $v$ in the case that 
$v$ is not single valued (which entails either a Kutta type condition for the velocity or a vanishing density perturbation on the cut), in initial and final times the following equation must be satisfied:

$$
\frac{\mathrm{d} v}{\mathrm{~d} t}=\frac{1}{2} \boldsymbol{v}^{2}-w .
$$

Finally we have to calculate the variation with respect to both $\chi$ and $\eta$, this will lead us to the following results:

$$
\begin{aligned}
\delta_{\chi} A= & \int \mathrm{d}^{3} x \mathrm{~d} t \delta \chi\left[\frac{\partial(\rho \alpha)}{\partial t}+\nabla \cdot(\rho \alpha \boldsymbol{v})-\nabla \eta \cdot \boldsymbol{J}\right] \\
& +\int \mathrm{d} t \oint \mathrm{d} \boldsymbol{S} \cdot\left[\frac{\boldsymbol{B}}{4 \pi} \times \nabla \eta-\boldsymbol{v} \rho \alpha\right] \delta \chi \\
& +\int \mathrm{d} t \int \mathrm{d} \boldsymbol{\Sigma} \cdot\left[\frac{\boldsymbol{B}}{4 \pi} \times \nabla \eta-\boldsymbol{v} \rho \alpha\right][\delta \chi] \\
& -\left.\int \mathrm{d}^{3} x \rho \alpha \delta \chi\right|_{t_{0}} ^{t_{1}}, \\
\delta_{\eta} A= & \int \mathrm{d}^{3} x \mathrm{~d} t \delta \eta\left[\frac{\partial(\rho \beta)}{\partial t}+\nabla \cdot(\rho \beta \boldsymbol{v})+\nabla \chi \cdot \boldsymbol{J}\right] \\
& +\int \mathrm{d} t \oint \mathrm{d} \boldsymbol{S} \cdot\left[\nabla \chi \times \frac{B}{4 \pi}-\boldsymbol{v} \rho \beta\right] \delta \eta \\
& +\int \mathrm{d} t \int \mathrm{d} \boldsymbol{\Sigma} \cdot\left[\nabla \chi \times \frac{B}{4 \pi}-\boldsymbol{v} \rho \beta\right][\delta \eta] \\
& -\left.\int \mathrm{d}^{3} x \rho \beta \delta \eta\right|_{t_{0}} ^{t_{1}} .
\end{aligned}
$$

Provided that the correct temporal and boundary conditions are met with respect to the variations $\delta \chi$ and $\delta \eta$ on the domain boundary and on the cuts in the case that some (or all) of the relevant functions are non-single-valued, we obtain the following set of equations:

$$
\frac{\mathrm{d} \alpha}{\mathrm{d} t}=\frac{\nabla \eta \cdot J}{\rho}, \quad \frac{\mathrm{d} \beta}{\mathrm{d} t}=-\frac{\nabla \chi \cdot J}{\rho},
$$

in which the continuity equation (2.3) was taken into account. By correct temporal conditions we mean that both $\delta \eta$ and $\delta \chi$ vanish at initial and final times. As for boundary conditions which are sufficient to make the boundary term vanish one can consider the case that the boundary is at infinity and both $\boldsymbol{B}$ and $\rho$ vanish. Another possibility is that the boundary is impermeable and perfectly conducting. A sufficient condition for the integral over the 'cuts' to vanish is to use variations $\delta \eta$ and $\delta \chi$ which are single valued. It can be shown that $\chi$ can always be taken to be single valued, hence taking $\delta \chi$ to be single valued is no restriction at all. In some topologies $\eta$ is not single valued and in those cases a single-valued restriction on $\delta \eta$ is sufficient to make the cut term null. 
Finally we take a variational derivative with respect to the entropy $s$,

$$
\begin{aligned}
\delta_{s} A= & \int \mathrm{d}^{3} x \mathrm{~d} t \delta s\left[\frac{\partial(\rho \sigma)}{\partial t}+\nabla \cdot(\rho \sigma \boldsymbol{v})-\rho T\right] \\
& +\int \mathrm{d} t \oint \mathrm{d} S \cdot \rho \sigma \boldsymbol{v} \delta s-\left.\int \mathrm{d}^{3} x \rho \sigma \delta s\right|_{t_{0}} ^{t_{1}},
\end{aligned}
$$

in which the temperature is $T=\partial \varepsilon / \partial s$. We notice that according to (3.6), $\sigma$ is single valued and hence no cuts are needed. Taking into account the continuity equation (2.3) we obtain for locations in which the density $\rho$ is not null the result that

$$
\frac{\mathrm{d} \sigma}{\mathrm{d} t}=T,
$$

provided that $\delta_{s} A$ vanished for an arbitrary $\delta s$.

\subsection{Euler's equations}

We shall now show that a velocity field given by (3.6), such that the equations for $\alpha, \beta, \chi, \eta, v, \sigma, s$ satisfy the corresponding equations (3.3), (3.8), (3.11a,b), (3.13) must satisfy Euler's equations. Let us calculate the material derivative of $\boldsymbol{v}$ as follows:

$$
\frac{\mathrm{d} v}{\mathrm{~d} t}=\frac{\mathrm{d} \nabla v}{\mathrm{~d} t}+\frac{\mathrm{d} \alpha}{\mathrm{d} t} \nabla \chi+\alpha \frac{\mathrm{d} \nabla \chi}{\mathrm{d} t}+\frac{\mathrm{d} \beta}{\mathrm{d} t} \nabla \eta+\beta \frac{\mathrm{d} \nabla \eta}{\mathrm{d} t}+\frac{\mathrm{d} \sigma}{\mathrm{d} t} \nabla s+\sigma \frac{\mathrm{d} \nabla s}{\mathrm{~d} t} .
$$

It can be easily shown that

$$
\begin{gathered}
\frac{\mathrm{d} \nabla v}{\mathrm{~d} t}=\nabla \frac{\mathrm{d} v}{\mathrm{~d} t}-\nabla v_{k} \frac{\partial v}{\partial x_{k}}=\nabla\left(\frac{1}{2} v^{2}-w\right)-\nabla v_{k} \frac{\partial v}{\partial x_{k}} \\
\frac{\mathrm{d} \nabla \eta}{\mathrm{d} t}=\nabla \frac{\mathrm{d} \eta}{\mathrm{d} t}-\nabla v_{k} \frac{\partial \eta}{\partial x_{k}}=-\nabla v_{k} \frac{\partial \eta}{\partial x_{k}} \\
\frac{\mathrm{d} \nabla \chi}{\mathrm{d} t}=\nabla \frac{\mathrm{d} \chi}{\mathrm{d} t}-\nabla v_{k} \frac{\partial \chi}{\partial x_{k}}=-\nabla v_{k} \frac{\partial \chi}{\partial x_{k}} \\
\frac{\mathrm{d} \nabla s}{\mathrm{~d} t}=\nabla \frac{\mathrm{d} s}{\mathrm{~d} t}-\nabla v_{k} \frac{\partial s}{\partial x_{k}}=-\nabla v_{k} \frac{\partial s}{\partial x_{k}}
\end{gathered}
$$

In which $x_{k}$ is a Cartesian coordinate and a summation convention is assumed. Inserting the results from (3.15) and (3.3) into (3.14) yields

$$
\begin{aligned}
\frac{\mathrm{d} \boldsymbol{v}}{\mathrm{d} t}= & -\nabla v_{k}\left(\frac{\partial v}{\partial x_{k}}+\alpha \frac{\partial \chi}{\partial x_{k}}+\beta \frac{\partial \eta}{\partial x_{k}}+\sigma \frac{\partial s}{\partial x_{k}}\right)+\nabla\left(\frac{1}{2} \boldsymbol{v}^{2}-w\right)+T \nabla s \\
& +\frac{1}{\rho}((\nabla \eta \cdot J) \nabla \chi-(\nabla \chi \cdot J) \nabla \eta) \\
= & -\nabla v_{k} v_{k}+\nabla\left(\frac{1}{2} \boldsymbol{v}^{2}-w\right)+T \nabla s+\frac{1}{\rho} \boldsymbol{J} \times(\nabla \chi \times \nabla \eta) \\
= & -\frac{\nabla p}{\rho}+\frac{1}{\rho} \boldsymbol{J} \times \boldsymbol{B} .
\end{aligned}
$$


In which we have used both (3.6) and (3.4) in the above derivation. This of course proves that the non-barotropic Euler equations can be derived from the action given in (3.1), and hence all the equations of non-barotropic MHD can be derived from the above action without restricting the variations in any way except on the relevant boundaries and cuts.

\subsection{Local non-barotropic cross-helicity}

The function $v$, whose material derivative is given in (3.8), can be multiple valued because only its gradient appears in the velocity (3.6). However, the discontinuity, [v], of $v$ is conserved,

$$
\frac{\mathrm{d}[v]}{\mathrm{d} t}=0,
$$

since the terms on the right-hand side of (3.8) describe physical quantities and hence are single valued. A similar equation also holds for barotropic fluid dynamics and barotropic MHD (Yahalom \& Lynden-Bell 2008; Yahalom 2010, 2013a).

We now substitute the expressions for $\boldsymbol{B}$ and $\boldsymbol{v}$ given by (3.4) and (3.6), respectively, into the formula $H_{C} \equiv \int \boldsymbol{B} \cdot \boldsymbol{v} \mathrm{d}^{3} x$ for the cross-helicity (see (1.1)) to obtain

$$
H_{C}=\int \mathrm{d} \Phi[v]+\int \mathrm{d} \Phi \oint \sigma \mathrm{d} s,
$$

where the closed line integral is taken along a magnetic field line. Furthermore, $\mathrm{d} \Phi=$ $\boldsymbol{B} \cdot \mathrm{d} \boldsymbol{S}=(\nabla \chi \times \nabla \eta) \cdot \mathrm{d} \boldsymbol{S}=\mathrm{d} \chi \mathrm{d} \eta$ is a magnetic flux element which is comoving as governed by (2.1) and $\mathrm{d} S$ is an infinitesimal area element. Although the cross-helicity is not conserved for non-barotropic flows, inspection of the right-hand side of (3.18) reveals that it is made of a sum of two terms. One term is conserved, as both $\mathrm{d} \Phi$ and $[v]$ are comoving, and the other is not. This suggests the following definition for the non-barotropic cross-helicity:

$$
H_{C N B} \equiv \int \mathrm{d} \Phi[v]=H_{C}-\int \mathrm{d} \Phi \oint \sigma \mathrm{d} s .
$$

It can be written in the more conventional form,

$$
H_{C N B}=\int \boldsymbol{B} \cdot \boldsymbol{v}_{t} \mathrm{~d}^{3} x,
$$

in which the topological velocity field is defined as

$$
\boldsymbol{v}_{t}=\boldsymbol{v}-\sigma \nabla s .
$$

It should be noted that $H_{C N B}$ is conserved even for an MHD not satisfying the Sakurai topological constraint given in (3.4), provided that we have a field $\sigma$ satisfying the equation $\mathrm{d} \sigma / \mathrm{d} t=T$. This can be verified by direct derivation using only the equation of motion and the sigma equation. Thus the non-barotropic cross-helicity conservation law,

$$
\frac{\mathrm{d} H_{C N B}}{\mathrm{~d} t}=0,
$$

is more general than the variational principle described by (3.50) as follows from a direct computation using (2.1) and (2.3)-(2.5). Also note that, for a constant specific 
entropy $s$, we obtain $H_{C N B}=H_{C}$ and the non-barotropic cross-helicity reduces to the standard barotropic cross-helicity. The local form of (3.22) describing the evolution of $H_{C N B}$ per unit volume was described by (Webb et al. 2014a,b). To conclude, we introduce also a local topological conservation law in the spirit of Yahalom (Yahalom 2013a) which is the non-barotropic cross-helicity per unit of magnetic flux. This quantity which is equal to the discontinuity, $[v]$, of $v$ is conserved and can be written as a sum of the barotropic cross-helicity per unit flux and the closed line integral of $s \mathrm{~d} \sigma$ along a magnetic field line, namely

$$
[\nu]=\frac{\mathrm{d} H_{C N B}}{\mathrm{~d} \Phi}=\frac{\mathrm{d} H_{C}}{\mathrm{~d} \Phi}+\oint s \mathrm{~d} \sigma .
$$

\subsection{Simplified action}

The reader of this paper might argue here that the paper is misleading. The authors have declared that they are going to present a simplified action for non-barotropic MHD, instead they added six more functions $\alpha, \beta, \chi, \eta, \nu, \sigma$ to the standard set $\boldsymbol{B}, \boldsymbol{v}, \rho, s$ ! In the following we show that this is not so and the action given in equation (3.1) in a form suitable for a pedagogic presentation that can indeed be simplified. It is easy to show that the Lagrangian density appearing in (3.1) can be written in the form

$$
\begin{aligned}
\mathcal{L}= & -\rho\left[\frac{\partial v}{\partial t}+\alpha \frac{\partial \chi}{\partial t}+\beta \frac{\partial \eta}{\partial t}+\sigma \frac{\partial s}{\partial t}+\varepsilon(\rho, s)\right] \\
& +\frac{1}{2} \rho\left[(\boldsymbol{v}-\hat{\boldsymbol{v}})^{2}-(\hat{\boldsymbol{v}})^{2}\right] \\
& +\frac{1}{8 \pi}\left[(\boldsymbol{B}-\hat{\boldsymbol{B}})^{2}-(\hat{\boldsymbol{B}})^{2}\right]+\frac{\partial(v \rho)}{\partial t}+\nabla \cdot(v \rho \boldsymbol{v}),
\end{aligned}
$$

in which $\hat{\boldsymbol{v}}$ is a shorthand notation for $\nabla v+\alpha \nabla \chi+\beta \nabla \eta+\sigma \nabla s$ (see (3.6)) and $\hat{\boldsymbol{B}}$ is a shorthand notation for $\nabla \chi \times \nabla \eta$ (see (3.4)). Thus $\mathcal{L}$ has four contributions as follows:

$$
\begin{gathered}
\mathcal{L}=\hat{\mathcal{L}}+\mathcal{L}_{v}+\mathcal{L}_{B}+\mathcal{L}_{\text {boundary }}, \\
\hat{\mathcal{L}} \equiv-\rho\left[\frac{\partial v}{\partial t}+\alpha \frac{\partial \chi}{\partial t}+\beta \frac{\partial \eta}{\partial t}+\sigma \frac{\partial s}{\partial t}+\varepsilon(\rho, s)\right. \\
\left.+\frac{1}{2}(\nabla v+\alpha \nabla \chi+\beta \nabla \eta+\sigma \nabla s)^{2}\right] \\
-\frac{1}{8 \pi}(\nabla \chi \times \nabla \eta)^{2}, \\
\mathcal{L}_{v} \equiv \frac{1}{2} \rho(\boldsymbol{v}-\hat{\boldsymbol{v}})^{2}, \\
\mathcal{L}_{B} \equiv \frac{1}{8 \pi}(\boldsymbol{B}-\hat{\boldsymbol{B}})^{2} \\
\mathcal{L}_{\text {boundary }} \equiv \frac{\partial(v \rho)}{\partial t}+\nabla \cdot(v \rho \boldsymbol{v}) .
\end{gathered}
$$


The only term containing $\boldsymbol{v}$ is $\mathcal{L}_{v}\left(\mathcal{L}_{\text {boundary }}\right.$ also depends on $\boldsymbol{v}$ but being a boundary term in space and time it does not contribute to the derived equations), it can easily be seen that this term will lead, after we nullify the variational derivative with respect to $\boldsymbol{v}$, to (3.6) but will otherwise have no contribution to other variational derivatives. Similarly the only term containing $B$ is $\mathcal{L}_{B}$ and it can easily be seen that this term will lead, after we nullify the variational derivative, to (3.4), but will have no contribution to other variational derivatives. Also notice that the term $\mathcal{L}_{\text {boundary }}$ contains only complete partial derivatives and thus can not contribute to the equations, although it can change the boundary conditions. Hence we see that (3.3), (3.8), (3.11a,b) and (3.13) can be derived using the Lagrangian density,

$$
\begin{aligned}
& \hat{\mathcal{L}} {[\alpha, \beta, \chi, \eta, \nu, \rho, \sigma, s]=-\rho\left[\frac{\partial v}{\partial t}+\alpha \frac{\partial \chi}{\partial t}+\beta \frac{\partial \eta}{\partial t}\right.} \\
&\left.+\sigma \frac{\partial s}{\partial t}+\varepsilon(\rho, s)+\frac{1}{2}(\nabla v+\alpha \nabla \chi+\beta \nabla \eta+\sigma \nabla s)^{2}\right] \\
&-\frac{1}{8 \pi}(\nabla \chi \times \nabla \eta)^{2}
\end{aligned}
$$

in which $\hat{\boldsymbol{v}}$ replaces $\boldsymbol{v}$ and $\hat{\boldsymbol{B}}$ replaces $\boldsymbol{B}$ in the relevant equations. Furthermore, after integrating the eight equations (3.3), (3.8), (3.11a,b), (3.13) we can insert the potentials $\alpha, \beta, \chi, \eta, v, \sigma, s$ into (3.6) and (3.4) to obtain the physical quantities $\boldsymbol{v}$ and $\boldsymbol{B}$. Hence, the general non-barotropic magnetohydrodynamic problem is reduced from eight equations (2.1), (2.3), (2.4), (2.5) and the additional constraint (2.2), to a problem of eight first-order (in the temporal derivative) unconstrained equations. Moreover, the entire set of equations can be derived from the Lagrangian density $\hat{\mathcal{L}}$.

\subsection{Further simplification}

\subsubsection{Elimination of variables}

Let us now look at the last three equations of (3.3) (Yahalom 2016a,b). Those describe three comoving quantities which can be written in terms of the generalized Clebsch form given in (3.6) as follows:

$$
\left.\begin{array}{l}
\frac{\partial \chi}{\partial t}+(\nabla v+\alpha \nabla \chi+\beta \nabla \eta+\sigma \nabla s) \cdot \nabla \chi=0 \\
\frac{\partial \eta}{\partial t}+(\nabla v+\alpha \nabla \chi+\beta \nabla \eta+\sigma \nabla s) \cdot \nabla \eta=0 \\
\frac{\partial s}{\partial t}+(\nabla v+\alpha \nabla \chi+\beta \nabla \eta+\sigma \nabla s) \cdot \nabla s=0
\end{array}\right\} .
$$

Those are algebraic equations for $\alpha, \beta, \sigma$ which can be solved such that $\alpha, \beta, \sigma$ can be written as functionals of $\chi, \eta, v, s$ resulting eventually in the description of non-barotropic MHD in terms of five functions, $v, \rho, \chi, \eta, s$. Let us introduce the notation

$$
\alpha_{i} \equiv(\alpha, \beta, \sigma), \chi_{i} \equiv(\chi, \eta, s), k_{i} \equiv-\frac{\partial \chi_{i}}{\partial t}-\nabla \nu \cdot \nabla \chi_{i},
$$

$i \in(1,2,3)$. In terms of the above notation (3.27) takes the form

$$
k_{i}=\alpha_{j} \nabla \chi_{i} \cdot \nabla \chi_{j}, \quad j \in(1,2,3)
$$


in which the Einstein summation convention is assumed. Let us define the matrix

$$
A_{i j} \equiv \nabla \chi_{i} \cdot \nabla \chi_{j}
$$

where obviously this matrix is symmetric since $A_{i j}=A_{j i}$. Hence (3.29) takes the form

$$
k_{i}=A_{i j} \alpha_{j}, \quad j \in(1,2,3)
$$

Provided that the matrix $A_{i j}$ is not singular it has an inverse $A_{i j}^{-1}$ which can be written as

$$
A_{i j}^{-1}=|A|^{-1}\left(\begin{array}{ccc}
A_{22} A_{33}-A_{23}^{2} & A_{13} A_{23}-A_{12} A_{33} & A_{12} A_{23}-A_{13} A_{22} \\
A_{13} A_{23}-A_{12} A_{33} & A_{11} A_{33}-A_{13}^{2} & A_{12} A_{13}-A_{11} A_{23} \\
A_{12} A_{23}-A_{13} A_{22} & A_{12} A_{13}-A_{11} A_{23} & A_{11} A_{22}-A_{12}^{2}
\end{array}\right)
$$

in which the determinant $|A|$ is given by the following equation:

$$
|A|=A_{11} A_{22} A_{33}-A_{11} A_{23}^{2}-A_{22} A_{13}^{2}-A_{33} A_{12}^{2}+2 A_{12} A_{13} A_{23} .
$$

In terms of the above equations the $\alpha_{i}$ can be calculated as functionals of $\chi_{i}, v$ as follows:

$$
\alpha_{i}\left[\chi_{i}, v\right]=A_{i j}^{-1} k_{j}
$$

The velocity equation (3.6) can now be written as

$$
\begin{aligned}
v & =\nabla v+\alpha_{i} \nabla \chi_{i}=\nabla v+A_{i j}^{-1} k_{j} \nabla \chi_{i} \\
& =\nabla v-A_{i j}^{-1} \nabla \chi_{i}\left(\frac{\partial \chi_{j}}{\partial t}+\nabla v \cdot \nabla \chi_{j}\right) .
\end{aligned}
$$

Provided that the $\chi_{i}$ is a coordinate basis in three dimensions, we may write

$$
\nabla v=\nabla \chi_{n} \frac{\partial v}{\partial \chi_{n}}, \quad n \in(1,2,3)
$$

Inserting (3.36) into (3.35) we obtain

$$
\boldsymbol{v}=-A_{i j}^{-1} \nabla \chi_{i} \frac{\partial \chi_{j}}{\partial t}
$$

where in the above $\delta_{i n}$ is a Kronecker delta. Thus the velocity $v\left[\chi_{i}\right]$ is a functional of $\chi_{i}$ only and is independent of $v$. 


\subsection{Lagrangian density and variational analysis}

Let us now rewrite the Lagrangian density $\hat{\mathcal{L}}\left[\chi_{i}, v, \rho\right]$ given in (3.26) in terms of the new variables as

$$
\begin{aligned}
\hat{\mathcal{L}}\left[\chi_{i}, v, \rho\right]= & -\rho\left[\frac{\partial v}{\partial t}+\alpha_{k}\left[\chi_{i}, v\right] \frac{\partial \chi_{k}}{\partial t}+\varepsilon\left(\rho, \chi_{3}\right)+\frac{1}{2} \boldsymbol{v}\left[\chi_{i}\right]^{2}\right] \\
& -\frac{1}{8 \pi}\left(\nabla \chi_{1} \times \nabla \chi_{2}\right)^{2} .
\end{aligned}
$$

Let us calculate the variational derivative of $\hat{\mathcal{L}}\left[\chi_{i}, \nu, \rho\right]$ with respect to $\chi_{i}$, this will result in

$$
\begin{aligned}
\delta_{\chi_{i}} \hat{\mathcal{L}}= & -\rho\left[\delta_{\chi_{i}} \alpha_{k} \frac{\partial \chi_{k}}{\partial t}+\alpha_{\underline{i}} \frac{\partial \delta \chi_{i}}{\partial t}+\delta_{\chi_{i}} \varepsilon\left(\rho, \chi_{3}\right)+\delta_{\chi_{i}} \boldsymbol{v} \cdot \boldsymbol{v}\right] \\
& -\frac{\boldsymbol{B}}{4 \pi} \cdot \delta_{\chi_{i}}\left(\nabla \chi_{1} \times \nabla \chi_{2}\right)
\end{aligned}
$$

in which the summation convention is not applied if the index is underlined. However, due to (3.35) we may write

$$
\delta_{\chi_{i}} v=\delta_{\chi_{i}} \alpha_{k} \nabla \chi_{k}+\alpha_{\underline{i}} \nabla \delta \chi_{\underline{i}} .
$$

Inserting (3.40) into (3.39) and rearranging the terms we obtain

$$
\begin{aligned}
\delta_{\chi_{i}} \hat{\mathcal{L}}= & -\rho\left[\delta_{\chi_{i}} \alpha_{k}\left(\frac{\partial \chi_{k}}{\partial t}+\boldsymbol{v} \cdot \nabla \chi_{k}\right)+\alpha_{\underline{i}}\left(\frac{\partial \delta \chi_{\underline{i}}}{\partial t}+\boldsymbol{v} \cdot \nabla \delta \chi_{\underline{i}}\right)+\delta_{\chi_{i}} \varepsilon\left(\rho, \chi_{3}\right)\right] \\
& -\frac{\boldsymbol{B}}{4 \pi} \cdot \delta_{\chi_{i}}\left(\nabla \chi_{1} \times \nabla \chi_{2}\right) .
\end{aligned}
$$

Now by construction $v$ satisfies (3.27) and hence $\partial \chi_{k} / \partial t+\boldsymbol{v} \cdot \nabla \chi_{k}=0$, this leads to

$$
\delta_{\chi_{i}} \hat{\mathcal{L}}=-\rho\left[\alpha_{\underline{i}} \frac{\mathrm{d} \delta \chi_{\underline{i}}}{\mathrm{~d} t}+\delta_{\chi_{i}} \varepsilon\left(\rho, \chi_{3}\right)\right]-\frac{\boldsymbol{B}}{4 \pi} \cdot \delta_{\chi_{i}}\left(\nabla \chi_{1} \times \nabla \chi_{2}\right) .
$$

From now on the derivation proceeds as in (3.9), (3.10), (3.12) resulting in $(3.11 a, b)$, (3.13) and will not be repeated. The difference is that now $\alpha, \beta$ and $\sigma$ are not independent quantities, rather they depend through (3.34) on the derivatives of $\chi_{i}, v$. Thus, (3.9), (3.10), (3.12) are not first-order equations in time but are second-order equations. Now let us calculate the variational derivative with respect to $v$, this will result in the expression

$$
\delta_{v} \hat{\mathcal{L}}=-\rho\left[\frac{\partial \delta \nu}{\partial t}+\delta_{v} \alpha_{n} \frac{\partial \chi_{n}}{\partial t}\right] .
$$

However, $\delta_{\nu} \alpha_{k}$ can be calculated from (3.34) as follows:

$$
\delta_{\nu} \alpha_{n}=A_{n j}^{-1} \delta_{\nu} k_{j}=-A_{n j}^{-1} \nabla \delta v \cdot \nabla \chi_{j} .
$$


Inserting the above equation into (3.43) gives

$$
\begin{aligned}
\delta_{v} \hat{\mathcal{L}} & =-\rho\left[\frac{\partial \delta v}{\partial t}-A_{n j}^{-1} \nabla \chi_{j} \frac{\partial \chi_{n}}{\partial t} \cdot \nabla \delta v\right] \\
& =-\rho\left[\frac{\partial \delta v}{\partial t}+\boldsymbol{v} \cdot \nabla \delta v\right]=-\rho \frac{\mathrm{d} \delta v}{\mathrm{~d} t} .
\end{aligned}
$$

The above equation can be put to the form,

$$
\delta_{v} \hat{\mathcal{L}}=\delta v\left[\frac{\partial \rho}{\partial t}+\nabla \cdot(\rho \boldsymbol{v})\right]-\frac{\partial(\rho \delta v)}{\partial t}-\nabla \cdot(\rho \boldsymbol{v} \delta v) .
$$

This obviously leads to the continuity equation (2.3) and some boundary terms in space and time. The variational derivative with respect to $\rho$ is trivial and the analysis is identical to the one in (3.7), leading to (3.8). To conclude this subsection let us summarize the equations of non-barotropic MHD as follows:

$$
\left.\begin{array}{c}
\frac{\mathrm{d} v}{\mathrm{~d} t}=\frac{1}{2} \boldsymbol{v}^{2}-w, \frac{\partial \rho}{\partial t}+\nabla \cdot(\rho \boldsymbol{v})=0, \\
\frac{\mathrm{d} \sigma}{\mathrm{d} t}=T, \quad \frac{\mathrm{d} \alpha}{\mathrm{d} t}=\frac{\nabla \eta \cdot J}{\rho}, \quad \frac{\mathrm{d} \beta}{\mathrm{d} t}=-\frac{\nabla \chi \cdot J}{\rho}
\end{array}\right\}
$$

in which $\alpha, \beta, \sigma, v$ are functionals of $\chi, \eta, s, v$ as described above. It is easy to show as in (3.16) that those variational equations are equivalent to the physical equations.

\subsection{Lagrangian density in explicit form}

Let us put the Lagrangian density of (3.38) in a slightly more explicit form. First us look at the term $v^{2}$,

$$
\begin{aligned}
\boldsymbol{v}^{2} & =A_{i j}^{-1} \nabla \chi_{i} \frac{\partial \chi_{j}}{\partial t} A_{m n}^{-1} \nabla \chi_{m} \frac{\partial \chi_{n}}{\partial t} \\
& =A_{i j}^{-1} A_{m n}^{-1} A_{i m} \frac{\partial \chi_{j}}{\partial t} \frac{\partial \chi_{n}}{\partial t}=A_{j n}^{-1} \frac{\partial \chi_{j}}{\partial t} \frac{\partial \chi_{n}}{\partial t},
\end{aligned}
$$

where in the above we use (3.37) and (3.30). Next let us look at the expression

$$
\begin{aligned}
\alpha_{k}\left[\chi_{i}, \nu\right] \frac{\partial \chi_{k}}{\partial t} & =A_{k j}^{-1} k_{j} \frac{\partial \chi_{k}}{\partial t}=-\left(\frac{\partial \chi_{j}}{\partial t}+\nabla v \cdot \nabla \chi_{j}\right) A_{k j}^{-1} \frac{\partial \chi_{k}}{\partial t} \\
& =-A_{j k}^{-1} \frac{\partial \chi_{j}}{\partial t} \frac{\partial \chi_{k}}{\partial t}-\frac{\partial v}{\partial \chi_{m}} \frac{\partial \chi_{m}}{\partial t} .
\end{aligned}
$$

Inserting (3.48) and (3.49) into (3.38) leads to a Lagrangian density of a more standard quadratic form,

$$
\begin{aligned}
\hat{\mathcal{L}}\left[\chi_{i}, v, \rho\right]= & \rho\left[\frac{1}{2} A_{j n}^{-1} \frac{\partial \chi_{j}}{\partial t} \frac{\partial \chi_{n}}{\partial t}+\frac{\partial v}{\partial \chi_{m}} \frac{\partial \chi_{m}}{\partial t}-\frac{\partial v}{\partial t}-\varepsilon\left(\rho, \chi_{3}\right)\right] \\
& -\frac{1}{8 \pi}\left(\nabla \chi_{1} \times \nabla \chi_{2}\right)^{2} .
\end{aligned}
$$


We now define the metric $g_{j n}=A_{j n}^{-1}$ and obtain the geometrical Lagrangian,

$$
\begin{aligned}
\hat{\mathcal{L}}\left[\chi_{i}, \nu, \rho\right]= & \rho\left[\frac{1}{2} g_{j n} \frac{\partial \chi_{j}}{\partial t} \frac{\partial \chi_{n}}{\partial t}+\frac{\partial v}{\partial \chi_{m}} \frac{\partial \chi_{m}}{\partial t}-\frac{\partial v}{\partial t}-\varepsilon\left(\rho, \chi_{3}\right)\right] \\
& -\frac{1}{8 \pi}\left(\nabla \chi_{1} \times \nabla \chi_{2}\right)^{2} .
\end{aligned}
$$

The Lagrangian is thus composed of a geometric kinetic term which is quadratic in the temporal derivatives, a 'gyroscopic' term which is linear in the temporal derivative and a potential term which is independent of the temporal derivative.

\section{Noether current}

Let us assume that all the equations of motion and boundary conditions of non-barotropic MHD are satisfied. In this case we have, according to (3.26),

$$
\delta A=\int_{t_{1}}^{t_{2}} \mathrm{~d} t \int \mathrm{d}^{3} x \delta \hat{\mathcal{L}}=-\left.\int \mathrm{d}^{3} x \rho[\delta v+\alpha \delta \chi+\beta \delta \eta+\sigma \delta s]\right|_{t_{1}} ^{t_{2}} .
$$

For the current purpose it does not matter if $\alpha, \beta$ and $\sigma$ are independent variational variables or depend on other variational variables through (3.34). Now suppose that the variations $\delta v, \delta \chi, \delta \eta, \delta s$ are symmetry variations such that $\delta A=0$. In that case one obtains a conserved Noether current,

$$
\delta J=-\int \mathrm{d}^{3} x \rho[\delta v+\alpha \delta \chi+\beta \delta \eta+\sigma \delta s] .
$$

As the variations in the specific entropy $s$ will generally vary the specific internal energy term in the Lagrangian, we do not expect non-trivial entropy symmetry transformation, and the action will only be invariant for $\delta s=0$, hence

$$
\delta J=-\int \mathrm{d}^{3} x \rho[\delta v+\alpha \delta \chi+\beta \delta \eta] .
$$

\subsection{Lagrangian and Eulerian variations}

The value of a function $f$ can be modified by evaluating it at a different point in space, the difference between the new and old values would be

$$
f(\boldsymbol{x}+\boldsymbol{\xi})-f(\boldsymbol{x})=\boldsymbol{\xi} \cdot \nabla f,
$$

in which $\boldsymbol{x}$ is a coordinate vector and $\boldsymbol{\xi}$ is a displacement vector, the equality is correct to first order in $\xi$. Alternatively we can modify the value of a function by changing it to a different function $f^{\prime}$, in this case the difference between the new and old values would be

$$
\delta f=f^{\prime}(\boldsymbol{x})-f(\boldsymbol{x}) .
$$

For a small $\delta f$ this just the standard variation of variational analysis or an Eulerian variation. Finally we can do both, in the last case the difference between the new and 
old values would be

$$
\Delta f=f^{\prime}(\boldsymbol{x}+\xi)-f(\boldsymbol{x}),
$$

hence

$$
\Delta f=f^{\prime}(\boldsymbol{x}+\xi)-f(\boldsymbol{x}+\xi)+f(\boldsymbol{x}+\xi)-f(\boldsymbol{x}) .
$$

Keeping only first-order terms we obtain

$$
\Delta f=\delta f+\xi \cdot \nabla f \Rightarrow \delta f=\Delta f-\xi \cdot \nabla f
$$

in which $\Delta$ is a Lagrangian variation.

Now suppose that a specific function is connected to a fluid element in such a way that its value in space is determined only by fluid element location. And suppose that the fluid element is displaced as dictated by the flow. Such a function would be denoted a label of the flow and its material derivative would vanish. Moreover, for a label

$$
f^{\prime}(\boldsymbol{x}+\xi)=f(\boldsymbol{x}) \Rightarrow \Delta f=0,
$$

in order to change the value of a label in a certain point in space the fluid element must be displaced and another (with a different label value) must take its place. If follows from (4.8) that for a label

$$
\delta f=-\xi \cdot \nabla f .
$$

Now suppose we have a set of three labels $\tilde{\chi}_{i}$ such that

$$
\delta \tilde{\chi}_{i}=-\xi \cdot \nabla \tilde{\chi}_{i}=-\xi_{k} \frac{\partial \tilde{\chi}_{i}}{\partial x_{k}}
$$

in which we use the Einstein summation convention and $x_{k}$ are Cartesian coordinates. The inverse of the matrix $\partial \tilde{\chi}_{i} / \partial x_{k}$ is $\partial x_{k} / \partial \tilde{\chi}_{i}$ as

$$
\frac{\partial \tilde{\chi}_{i}}{\partial x_{k}} \frac{\partial x_{j}}{\partial \tilde{\chi}_{i}}=\delta_{k}^{j}
$$

where $\delta_{k}^{j}$ is a Kronecker delta. It thus follows that one can calculate the displacement vector $\xi$ as follows:

$$
\xi_{k}=-\frac{\partial x_{k}}{\partial \tilde{\chi}_{i}} \delta \tilde{\chi}_{i} \Rightarrow \boldsymbol{\xi}=-\frac{\partial \boldsymbol{r}}{\partial \tilde{\chi}_{i}} \delta \tilde{\chi}_{i}
$$

\subsection{Noether current for label symmetries}

We now study the form of the Noether current equation (4.3) for the case of label symmetry transformations. It is clear from (3.3) that $\chi, \eta$ can be taken to be labels. Hence we can 
write the conserved Noether current defined in (4.3) as

$$
\delta J=-\int \mathrm{d}^{3} x \rho[\Delta v-\xi \cdot \nabla v-\alpha \xi \cdot \nabla \chi-\beta \xi \cdot \nabla \eta],
$$

or using (3.6) as

$$
\delta J=\int \mathrm{d}^{3} x \rho[\xi \cdot(\boldsymbol{v}-\sigma \nabla s)-\Delta v] .
$$

We use the topological velocity defined in (3.21) to get

$$
\boldsymbol{v}_{t}=\nabla v+\alpha \nabla \chi+\beta \nabla \eta,
$$

and write

$$
\delta J=\int \mathrm{d}^{3} x \rho\left[\xi \cdot \boldsymbol{v}_{t}-\Delta v\right] .
$$

Suppose now that we are considering label symmetry transformations with the infinitesimal form $\tilde{\chi}_{i}+\delta \tilde{\chi}_{i}$, this type of transformation will induce a transformation on other functions ( $v$ as an example) which could be thought of as functions of labels, a transformation of the form

$$
\delta v=v\left(\tilde{\chi}_{i}+\delta \tilde{\chi}_{i}\right)-v\left(\tilde{\chi}_{i}\right)=\delta \tilde{\chi}_{i} \partial_{\tilde{\chi}_{i}} v=-\xi \cdot \nabla \tilde{\chi}_{i} \partial_{\tilde{\chi}_{i}} \nu=-\xi \cdot \nabla v .
$$

Hence by (4.8) we have

$$
\Delta v=0 .
$$

It follows that for an induced infinitesimal label transformation any function will transform as a label. From (4.19) it follows that the Noether current will take the following form for a symmetry label transformation:

$$
\delta J=\int \mathrm{d}^{3} x \rho \xi \cdot \boldsymbol{v}_{t}
$$

This Noether current form is identical to (47) of Yahalom (2017d) and (14) of Yahalom (2019b), which were derived from a Lagrangian variational principle. We note, however, that this form is limited to the case of label transformations and the general form given in (4.2) allows us to exploit larger symmetry groups. Next we will study some symmetry transformations of the action $A$, in order to do this we shall first introduce the load and metage quantities.

\section{Load and metage}

The following section follows closely similar sections in Yahalom \& Lynden-Bell (2008), Yahalom (2017a), Yahalom (2017d) and Yahalom (2019a). Consider a thin tube 
surrounding a magnetic field line, the magnetic flux contained within the tube is

$$
\Delta \Phi=\int \boldsymbol{B} \cdot \mathrm{d} \boldsymbol{S}
$$

and the mass contained with the tube is

$$
\Delta M=\int \rho \mathrm{d} \boldsymbol{l} \cdot \mathrm{d} \boldsymbol{S},
$$

in which $d l$ is a length element along the tube. Since the magnetic field lines move with the flow by virtue of (2.1) and (2.3), both the quantities $\Delta \Phi$ and $\Delta M$ are conserved, and since the tube is thin we may define the conserved magnetic load as

$$
\lambda=\frac{\Delta M}{\Delta \Phi}=\oint \frac{\rho}{B} \mathrm{~d} l
$$

in which the above integral is performed along the field line. Obviously the parts of the line which go out of the flow to regions in which $\rho=0$ have a null contribution to the integral. Notice that $\lambda$ is a single-valued function that can be measured in principle. Since $\lambda$ is conserved it satisfies the equation

$$
\frac{\mathrm{d} \lambda}{\mathrm{d} t}=0 .
$$

This can be viewed as a manifestation of the frozen-in law of $B / \rho$. By construction, surfaces of constant magnetic load move with the flow and contain magnetic field lines. Hence the gradient to such surfaces must be orthogonal to the field line,

$$
\nabla \lambda \cdot B=0 .
$$

Now consider an arbitrary comoving point on the magnetic field line and denote it by $i$, and consider an additional comoving point on the magnetic field line and denote it by $r$. The integral,

$$
\mu(r)=\int_{i}^{r} \frac{\rho}{B} \mathrm{~d} l+\mu(i),
$$

is also a conserved quantity which we may denote following Lynden-Bell \& Katz (1981) as the magnetic metage. Here $\mu(i)$ is an arbitrary number which can be chosen differently for each magnetic line. By construction we have

$$
\frac{\mathrm{d} \mu}{\mathrm{d} t}=0 .
$$

This can be viewed as another manifestation of the frozen-in law of $B / \rho$. Also it is easy to see that by differentiating along the magnetic field line we obtain

$$
\nabla \mu \cdot \boldsymbol{B}=\rho .
$$

Notice that $\mu$ will be generally a non-single-valued function; we will show later in this paper that symmetry to translations in $\mu$ will generate through Noether's theorem the conservation of the magnetic cross-helicity. 
At this point we have two comoving coordinates of flow, namely $\lambda, \mu$ obviously in a three-dimensional flow we also have a third coordinate. However, before defining the third coordinate we will find it useful to work not directly with $\lambda$ but with a function of $\lambda$. Now consider the magnetic flux within a surface of constant load $\Phi(\lambda)$. The magnetic flux is a conserved quantity and depends only on the load $\lambda$ of the surrounding surface. Now we define the quantity

$$
\chi=\frac{\Phi(\lambda)}{2 \pi} .
$$

Obviously $\chi$ satisfies the equations

$$
\frac{\mathrm{d} \chi}{\mathrm{d} t}=0, \quad B \cdot \nabla \chi=0 .
$$

Let us now define an additional comoving coordinate $\eta^{*}$, since $\nabla \mu$ is not orthogonal to the $B$ lines we can choose $\nabla \eta^{*}$ to be orthogonal to the $B$ lines and not in the direction of the $\nabla \chi$ lines - that is we choose $\eta^{*}$ not to depend only on $\chi$. Since both $\nabla \eta^{*}$ and $\nabla \chi$ are orthogonal to $\boldsymbol{B}, \boldsymbol{B}$ must take the form

$$
B=A \nabla \chi \times \nabla \eta^{*} .
$$

However, using (2.2) we have

$$
\nabla \cdot B=\nabla A \cdot\left(\nabla \chi \times \nabla \eta^{*}\right)=0,
$$

which implies that $A$ is a function of $\chi, \eta^{*}$. Now we can define a new comoving function $\eta$ such that

$$
\eta=\int_{0}^{\eta^{*}} A\left(\chi, \eta^{\prime *}\right) \mathrm{d} \eta^{\prime *}, \quad \frac{\mathrm{d} \eta}{\mathrm{d} t}=0 .
$$

In terms of this function we obtain the Sakurai (Euler potentials) presentation,

$$
B=\nabla \chi \times \nabla \eta .
$$

The density is now given by the Jacobian as follows:

$$
\rho=\nabla \mu \cdot(\nabla \chi \times \nabla \eta)=\frac{\partial(\chi, \eta, \mu)}{\partial(x, y, z)} .
$$

It can easily be shown using the fact that the labels are comoving, that the above forms of $\boldsymbol{B}$ and $\rho$ satisfy (2.1), (2.2) and (2.3) automatically.

We can now write a Lagrangian density in terms of the labels, in which $\rho$ is no longer an independent variational variable but rather a quantity dependent on $\mu$ through (5.15). The Lagrangian density of (3.26) takes the form,

$$
\begin{aligned}
& \hat{\mathcal{L}}[\alpha, \beta, \chi, \eta, \mu, \nu, \sigma, s]=-\frac{\partial(\chi, \eta, \mu)}{\partial(x, y, z)}\left[\frac{\partial v}{\partial t}+\alpha \frac{\partial \chi}{\partial t}+\beta \frac{\partial \eta}{\partial t}+\sigma \frac{\partial s}{\partial t}\right. \\
& \left.\quad+\varepsilon\left(\frac{\partial(\chi, \eta, \mu)}{\partial(x, y, z)}, s\right)+\frac{1}{2}(\nabla v+\alpha \nabla \chi+\beta \nabla \eta+\sigma \nabla s)^{2}\right]-\frac{1}{8 \pi}(\nabla \chi \times \nabla \eta)^{2} .
\end{aligned}
$$

Notice, however, that $\eta$ is defined in a non-unique way since one can redefine $\eta$, for example by performing the following transformation: $\eta \rightarrow \eta+f(\chi)$, in which $f(\chi)$ is an 
arbitrary function. The comoving coordinates $\chi, \eta$ serve as labels of the magnetic field lines. Moreover, the magnetic flux can be calculated as

$$
\Phi=\int \boldsymbol{B} \cdot \mathrm{d} \boldsymbol{S}=\int \mathrm{d} \chi \mathrm{d} \eta
$$

In the case that the surface integral is performed inside a load contour we obtain

$$
\Phi(\lambda)=\int_{\lambda} \mathrm{d} \chi \mathrm{d} \eta=\chi \int_{\lambda} \mathrm{d} \eta=\left\{\begin{array}{c}
\chi[\eta], \\
\chi\left(\eta_{\max }-\eta_{\min }\right) .
\end{array}\right.
$$

There are two cases involved. In one case the load surfaces are topological cylinders; in this case $\eta$ is not single valued and hence we obtain the upper value for $\Phi(\lambda)$. In a second case the load surfaces are topological spheres; in this case $\eta$ is single valued and has minimal $\eta_{\min }$ and maximal $\eta_{\max }$ values. Hence the lower value of $\Phi(\lambda)$ is obtained. For example in some cases $\eta$ is identical to twice the latitude angle $\theta$. In those cases $\eta_{\min }=0$ (the value at the 'north pole') and $\eta_{\max }=2 \pi$ (the value at the 'south pole').

Comparing the above equation with (5.9), we derive that $\eta$ can be either single valued or not single valued, and that its discontinuity across its cut in the non-single-valued case is $[\eta]=2 \pi$.

The triplet $\chi, \eta, \mu$ will suffice to label any fluid element in three dimensions. But for a non-barotropic flow there is also another possible label $s$ which is comoving according to (2.5). The question then arises of the relation of this label to the previous three. As one needs to make a choice regarding the preferred set of labels it seems that the physical ones are $\chi, \eta, s$, in which we use the surfaces on which the magnetic fields lie and the entropy, each label has an obvious physical interpretation. In this case we must look at $\mu$ as a function of $\chi, \eta, s$. If the magnetic field lines lie on the entropy surface then $\mu$ regains its status as an independent label. The density can now be written as

$$
\rho=\frac{\partial \mu}{\partial s} \frac{\partial(\chi, \eta, s)}{\partial(x, y, z)} .
$$

Now as $\mu$ can be defined for each magnetic field line separately according to (5.6), it is obvious that such a choice exists in which $\mu$ is a function of $s$ only. One may also think of the entropy $s$ as a function of $\chi, \eta, \mu$. However, if one changes $\mu$ in this case, this generally entails a change in $s$ and the symmetry described in (5.6) is lost in the action. In what follows we shall ignore the status of $s$ as a label and consider it as a variational variable which only attains a status of a label at the variational extremum.

\section{The labelling symmetry group and its subgroups}

It is obvious that the choice of fluid labels is quite arbitrary. However, when enforcing the $\chi, \eta, \mu$ coordinate system satisfying (5.15) the choice is restricted to $\tilde{\chi}, \tilde{\eta}, \tilde{\mu}$ such that

$$
\frac{\partial(\tilde{\chi}, \tilde{\eta}, \tilde{\mu})}{\partial(\chi, \eta, \mu)}=1 .
$$

Moreover, the Euler potential magnetic field representation,

$$
B=\nabla \chi \times \nabla \eta,
$$


reduces the choice further to

$$
\frac{\partial(\tilde{\chi}, \tilde{\eta})}{\partial(\chi, \eta)}=1
$$

We further notice that in the Eulerian variation principle approach the label symmetry cannot be realized unless it is coupled to the transformation of other variational variables, that is the label transformation induces transformation on $\alpha$ and $\beta$ as follows:

$$
\left.\begin{array}{l}
\tilde{\alpha}=\alpha \partial_{\tilde{\chi}} \chi+\beta \partial_{\tilde{\chi}} \eta \\
\tilde{\beta}=\alpha \partial_{\tilde{\eta}} \chi+\beta \partial_{\tilde{\eta}} \eta
\end{array}\right\}
$$

From (5.16) it follows that:

$$
\hat{\mathcal{L}}[\alpha, \beta, \chi, \eta, \mu, v, \sigma, s]-\hat{\mathcal{L}}[\tilde{\alpha}, \tilde{\beta}, \tilde{\chi}, \tilde{\eta}, \tilde{\mu}, \nu, \sigma, s]=0,
$$

hence the label transformation is a symmetry transformation. Now suppose that we consider $v$ as a function of the labels

$$
v(x, y, z, t)=\bar{v}(\chi, \eta, \mu, t)
$$

in that case replacing $(\chi, \eta, \mu) \rightarrow(\tilde{\chi}, \tilde{\eta}, \tilde{\mu})$ in the above equation will yield a different function $\tilde{v}$ of the coordinates such that

$$
\bar{v}(\tilde{\chi}, \tilde{\eta}, \tilde{\mu}, t)=\tilde{v}(x, y, z, t) .
$$

From this point of view, which we adopt in the current paper, symmetry can be only achieved if

$$
\int \mathrm{d}^{3} x[\hat{\mathcal{L}}[\alpha, \beta, \chi, \eta, \mu, \bar{v}(\chi, \eta, \mu, t), \sigma, s]-\hat{\mathcal{L}}[\tilde{\alpha}, \tilde{\beta}, \tilde{\chi}, \tilde{\eta}, \tilde{\mu}, \bar{v}(\tilde{\chi}, \tilde{\eta}, \tilde{\mu}, t), \sigma, s]]=0
$$

\subsection{Metage translations}

In what follows we consider the transformation (see also (5.6))

$$
\tilde{\chi}=\chi, \tilde{\eta}=\eta, \tilde{\mu}=\mu+a(\chi, \eta) .
$$

Hence $a$ is a label displacement which may be different for each magnetic field line, as the field line is closed one need not worry about edge difficulties. This transformation satisfies trivially the conditions (6.1), (6.3). If we take the infinitesimal symmetry transformation $\delta \mu=a, \delta \chi=\delta \eta=0$ we can calculate the associated fluid element displacement with 
this relabeling using (4.13) and (5.8), giving

$$
\boldsymbol{\xi}=-\frac{\partial \boldsymbol{r}}{\partial \mu} \delta \mu=-\delta \mu \frac{\boldsymbol{B}}{\rho} .
$$

Inserting (6.10) into (4.20) we obtain the conservation law,

$$
\delta J=\int \mathrm{d}^{3} x \rho \boldsymbol{v}_{t} \cdot \boldsymbol{\xi}=-\int \mathrm{d}^{3} x \delta \mu \boldsymbol{v}_{t} \cdot \boldsymbol{B} .
$$

In the simplest case we may take $\delta \mu$ to be a small constant, hence

$$
\delta J=-\delta \mu \int \mathrm{d}^{3} x \boldsymbol{v}_{t} \cdot \boldsymbol{B}=-\delta \mu H_{C N B},
$$

where $H_{C N B}$ is the non-barotropic global cross-helicity (Webb et al. 2014a; Yahalom $2017 b, c)$ defined as

$$
H_{C N B} \equiv \int \mathrm{d}^{3} x \boldsymbol{v}_{t} \cdot \boldsymbol{B}
$$

We thus obtain the conservation of non-barotropic cross-helicity using Noether's theorem and the symmetry group of metage translations. Of course one can perform a different translation on each magnetic field line, in this case one obtains

$$
\delta J=-\int \mathrm{d}^{3} x \delta \mu \boldsymbol{v}_{t} \cdot \boldsymbol{B}=-\int \mathrm{d} \chi \mathrm{d} \eta \delta \mu \oint_{\chi, \eta} \mathrm{d} \mu \rho^{-1} \boldsymbol{v}_{t} \cdot \boldsymbol{B} .
$$

Now since $\delta \mu$ is an arbitrary (small) function of $\chi, \eta$ it follows that

$$
I=\oint_{\chi, \eta} \mathrm{d} \mu \rho^{-1} \boldsymbol{v}_{t} \cdot \boldsymbol{B}
$$

is a conserved quantity for each magnetic field line. Along a magnetic field line the following equations hold:

$$
\mathrm{d} \mu=\nabla \mu \cdot \mathrm{d} r=\nabla \mu \cdot \hat{B} \mathrm{~d} r=\frac{\rho}{B} \mathrm{~d} r
$$

In the above, $\hat{B}$ is a unit vector in the magnetic field direction and (5.8) is used. Inserting (6.16) into (6.15) we obtain

$$
I=\oint_{\chi, \eta} \mathrm{d} r \boldsymbol{v}_{t} \cdot \hat{B}=\oint_{\chi, \eta} \mathrm{d} \boldsymbol{r} \cdot \boldsymbol{v}_{t},
$$

which is just the circulation of the topological velocity along the magnetic field lines. This quantity can be written in terms of the generalized Clebsch representation of the velocity equation (3.6) as

$$
I=\oint_{\chi, \eta} \mathrm{d} \boldsymbol{r} \cdot \boldsymbol{v}_{t}=\oint_{\chi, \eta} \mathrm{d} \boldsymbol{r} \cdot \nabla v=[v],
$$

where $[v]$ is the discontinuity of $v$. This was shown to be equal to the amount of non-barotropic cross-helicity per unit of magnetic flux in (3.23) (Yahalom 2017b,c),

$$
I=[v]=\frac{\mathrm{d} H_{C N B}}{\mathrm{~d} \Phi} .
$$




\subsection{Transformations of magnetic surfaces}

Consider the following transformations:

$$
\tilde{\eta}=\eta+\delta \eta(\chi, \eta), \quad \tilde{\chi}=\chi+\delta \chi(\chi, \eta), \quad \tilde{\mu}=\mu,
$$

in which $\delta \eta, \delta \chi$ are considered small in some sense. Inserting the above quantities into (6.3) and keeping only first-order terms we arrive at

$$
\partial_{\eta} \delta \eta+\partial_{\chi} \delta \chi=0
$$

This equation can be solved as follows:

$$
\delta \eta=\partial_{\chi} \delta f, \quad \delta \chi=-\partial_{\eta} \delta f,
$$

in which $\delta f=\delta f(\chi, \eta)$ is an arbitrary small function. In this case we obtain a particle displacement of the form,

$$
\begin{aligned}
\xi & =-\frac{\partial \boldsymbol{r}}{\partial \chi} \delta \chi-\frac{\partial \boldsymbol{r}}{\partial \eta} \delta \eta=-\frac{1}{\rho}(\nabla \eta \times \nabla \mu \delta \chi+\nabla \mu \times \nabla \chi \delta \eta) \\
& =\frac{\nabla \mu}{\rho} \times(\nabla \eta \delta \chi-\nabla \chi \delta \eta) .
\end{aligned}
$$

A special case that satisfies (6.21) is the case of a constant $\delta \chi$ and $\delta \eta$, those two independent displacements lead to the following two new topological conservation laws,

$$
\left.\begin{array}{l}
\delta J_{\chi}=\delta \chi \int \mathrm{d}^{3} x \boldsymbol{v}_{t} \cdot \nabla \mu \times \nabla \eta=\delta \chi H_{C N B \chi}, \\
\delta J_{\eta}=\delta \eta \int \mathrm{d}^{3} x \boldsymbol{v}_{t} \cdot \nabla \chi \times \nabla \mu=\delta \eta H_{C N B \eta},
\end{array}\right\}
$$

where the new non-barotropic global cross-helicities are defined as

$$
H_{C N B \chi} \equiv \int \mathrm{d}^{3} x \boldsymbol{v}_{t} \cdot \nabla \mu \times \nabla \eta, \quad H_{C N B \eta} \equiv \int \mathrm{d}^{3} x \boldsymbol{v}_{t} \cdot \nabla \chi \times \nabla \mu .
$$

We will find it useful to introduce the abstract 'magnetic fields' as follows:

$$
\boldsymbol{B}_{\chi} \equiv \nabla \mu \times \nabla \eta, \quad \boldsymbol{B}_{\eta} \equiv \nabla \chi \times \nabla \mu,
$$

in terms of which we obtain the new helicities in a more conventional form, i.e.

$$
H_{C N B \chi}=\int \mathrm{d}^{3} x \boldsymbol{v}_{t} \cdot \boldsymbol{B}_{\chi}, \quad H_{C N B \eta}=\int \mathrm{d}^{3} x \boldsymbol{v}_{t} \cdot \boldsymbol{B}_{\eta} .
$$

It is more plausible that those symmetries and conservation laws hold for magnetic field lines which lie on topological tori. In this case $\eta$ is non-single-valued (Yahalom \& Lynden-Bell 2008) and thus the translation in this direction resembles moving fluid elements along closed loops. Both those helicities suffer a topological interpretation in terms of the knottiness of the abstract magnetic field lines and the flow lines. Finally we remark that for barotropic MHD $\boldsymbol{v}_{t}$ can be replaced with $\boldsymbol{v}$. 


\section{Direct derivation}

Before continuing to discuss the possible applications of the topological constants of motion, we shall demonstrate that the generalized cross-helicities are indeed constant without relying on Noether's theorem.

\subsection{Direct derivation of the constancy of non-barotropic cross-helicity}

Taking the temporal derivative of the non-barotropic cross-helicity given in (6.13) we obtain,

$$
\frac{\mathrm{d} H_{C N B}}{\mathrm{~d} t}=\int \mathrm{d}^{3} x\left[\partial_{t} \boldsymbol{v}_{t} \cdot \boldsymbol{B}+\boldsymbol{v}_{t} \cdot \partial_{t} \boldsymbol{B}\right],
$$

where $\mathrm{d} / \mathrm{d} t$ is an ordinary temporal derivative, and we use the notation, $\partial_{t} \equiv \partial / \partial t$. Using (2.1) it follows that:

$$
\boldsymbol{v}_{t} \cdot \partial_{t} \boldsymbol{B}=\boldsymbol{v}_{t} \cdot \nabla \times(\boldsymbol{v} \times \boldsymbol{B})=\nabla \cdot\left((\boldsymbol{v} \times \boldsymbol{B}) \times \boldsymbol{v}_{t}\right)+(\boldsymbol{v} \times \boldsymbol{B}) \cdot \boldsymbol{\omega}_{t},
$$

in which we have used a standard identity of vector analysis and the definition,

$$
\omega_{t}=\nabla \times v_{t}=\omega-\nabla \sigma \times \nabla s,
$$

where $\boldsymbol{v}_{t}$ is defined in (3.21). Next we calculate

$$
\partial_{t} \boldsymbol{v}_{t} \cdot \boldsymbol{B}=\boldsymbol{B} \cdot \partial_{t}(\boldsymbol{v}-\sigma \nabla s)=\boldsymbol{B} \cdot\left(\partial_{t} \boldsymbol{v}-\partial_{t} \sigma \nabla s-\sigma \nabla \partial_{t} s\right)
$$

Taking into account Euler's equations (2.4) and the standard thermodynamic identities of (2.6) we have,

$$
\begin{aligned}
\partial_{t} \boldsymbol{v} & =-(\boldsymbol{v} \cdot \nabla) \boldsymbol{v}-\frac{1}{\rho} \nabla p(\rho, s)+\frac{(\nabla \times B) \times \boldsymbol{B}}{4 \pi \rho} \\
& =\boldsymbol{v} \times \boldsymbol{\omega}-\nabla\left(\frac{1}{2} \boldsymbol{v}^{2}\right)-\nabla w+T \nabla s+\frac{(\nabla \times B) \times \boldsymbol{B}}{4 \pi \rho} .
\end{aligned}
$$

Hence we have

$$
\boldsymbol{B} \cdot \partial_{t} \boldsymbol{v}=\boldsymbol{B} \cdot\left(\boldsymbol{v} \times \boldsymbol{\omega}-\nabla\left(\frac{1}{2} \boldsymbol{v}^{2}+w\right)+T \nabla s\right) .
$$

Taking into account (3.13) it follows that:

$$
-\partial_{t} \sigma \nabla s=(\boldsymbol{v} \cdot \nabla \sigma-T) \nabla s
$$

And taking into account (2.5) it follows that:

$$
-\sigma \nabla \partial_{t} s=\sigma \nabla(\boldsymbol{v} \cdot \nabla s)
$$

Inserting (7.6), (7.7) and (7.8) into (7.4) it follows that:

$$
\partial_{t} \boldsymbol{v}_{t} \cdot \boldsymbol{B}=\boldsymbol{B} \cdot\left(\boldsymbol{v} \times \boldsymbol{\omega}-\nabla\left(\frac{1}{2} \boldsymbol{v}^{2}+w\right)+(\boldsymbol{v} \cdot \nabla \sigma) \nabla s+\sigma \nabla(\boldsymbol{v} \cdot \nabla s)\right),
$$


hence

$$
\begin{aligned}
\partial_{t} \boldsymbol{v}_{t} \cdot \boldsymbol{B} & =\boldsymbol{B} \cdot\left(\boldsymbol{v} \times \boldsymbol{\omega}+\nabla\left(\sigma(\boldsymbol{v} \cdot \nabla s)-\frac{1}{2} \boldsymbol{v}^{2}-w\right)+(\boldsymbol{v} \cdot \nabla \sigma) \nabla s-(\boldsymbol{v} \cdot \nabla s) \nabla \sigma\right) \\
& =\boldsymbol{B} \cdot\left(\boldsymbol{v} \times \boldsymbol{\omega}+\nabla\left(\sigma(\boldsymbol{v} \cdot \nabla s)-\frac{1}{2} \boldsymbol{v}^{2}-w\right)+(\nabla \sigma \times \nabla s) \times \boldsymbol{v}\right) \\
& =\boldsymbol{B} \cdot\left(\boldsymbol{v} \times \boldsymbol{\omega}_{t}+\nabla\left(\sigma(\boldsymbol{v} \cdot \nabla s)-\frac{1}{2} \boldsymbol{v}^{2}-w\right)\right) .
\end{aligned}
$$

Combining (7.2) with (7.10) we arrive at the result,

$$
\begin{aligned}
\partial_{t} \boldsymbol{v}_{t} \cdot \boldsymbol{B}+\boldsymbol{v}_{t} \cdot \partial_{t} \boldsymbol{B} & =\nabla \cdot\left((\boldsymbol{v} \times \boldsymbol{B}) \times \boldsymbol{v}_{t}\right)+\boldsymbol{B} \cdot \boldsymbol{\nabla}\left(\sigma(\boldsymbol{v} \cdot \nabla s)-\frac{1}{2} \boldsymbol{v}^{2}-w\right) \\
& =\nabla \cdot\left[(\boldsymbol{v} \times \boldsymbol{B}) \times \boldsymbol{v}_{t}+\boldsymbol{B}\left(\sigma(\boldsymbol{v} \cdot \nabla s)-\frac{1}{2} \boldsymbol{v}^{2}-w\right)\right],
\end{aligned}
$$

in which we take into account (2.2). Inserting (7.11) into (7.1) and using Gauss' theorem we obtain a surface integral,

$$
\frac{\mathrm{d} H_{C N B}}{\mathrm{~d} t}=\oint \mathrm{d} \boldsymbol{S} \cdot\left[(\boldsymbol{v} \times \boldsymbol{B}) \times \boldsymbol{v}_{t}+\boldsymbol{B}\left(\sigma(\boldsymbol{v} \cdot \nabla s)-\frac{1}{2} \boldsymbol{v}^{2}-w\right)\right] .
$$

The surface integral encapsulates the volume for which the non-barotropic cross-helicity is calculated. If the surface is taken at infinity the magnetic fields vanish and thus

$$
\frac{\mathrm{d} H_{C N B}}{\mathrm{~d} t}=0,
$$

which means that $H_{C N B}$ is a constant of motion. We notice the complexity of the direct derivation with respect to the elegance and simplicity of the Noether theorem approach. However, obtaining the same result using different methods strengthens our confidence that no mathematical error was accidentally introduced.

\subsection{Direct derivation of the constancy of non-barotropic $\chi$ cross-helicity}

Taking the temporal derivative of the non-barotropic $\chi$ cross-helicity given in $(6.27 a, b)$ we obtain

$$
\frac{\mathrm{d} H_{C N B \chi}}{\mathrm{d} t}=\int \mathrm{d}^{3} x\left[\partial_{t} \boldsymbol{v}_{t} \cdot \boldsymbol{B}_{\chi}+\boldsymbol{v}_{t} \cdot \partial_{t} \boldsymbol{B}_{\chi}\right] .
$$

Let us calculate $\partial_{t} \boldsymbol{B}_{\chi}$ where $\boldsymbol{B}_{\chi}$ is defined in $(6.26 a, b)$ :

$$
B_{\chi}=\nabla \mu \times \nabla \eta
$$

It follows that

$$
\partial_{t} \boldsymbol{B}_{\chi}=\nabla \partial_{t} \mu \times \nabla \eta+\nabla \mu \times \nabla \partial_{t} \eta .
$$

Using (3.3) and (5.7) we obtain

$$
\begin{aligned}
\partial_{t} \boldsymbol{B}_{\chi} & =\nabla(-\boldsymbol{v} \cdot \nabla \mu) \times \nabla \eta+\nabla \mu \times \nabla(-\boldsymbol{v} \cdot \nabla \eta) \\
& =\nabla \times(\nabla \mu(\boldsymbol{v} \cdot \nabla \eta)-\nabla \eta(\boldsymbol{v} \cdot \nabla \mu)) \\
& =\nabla \times(\boldsymbol{v} \times(\nabla \mu \times \nabla \eta))=\nabla \times\left(\boldsymbol{v} \times \boldsymbol{B}_{\chi}\right),
\end{aligned}
$$


in which we used standard vector analysis identities. It thus follows that

$$
\boldsymbol{v}_{t} \cdot \partial_{t} \boldsymbol{B}_{\chi}=\boldsymbol{v}_{t} \cdot \nabla \times\left(\boldsymbol{v} \times \boldsymbol{B}_{\chi}\right)=\nabla \cdot\left(\left(\boldsymbol{v} \times \boldsymbol{B}_{\chi}\right) \times \boldsymbol{v}_{t}\right)+\left(\boldsymbol{v} \times \boldsymbol{B}_{\chi}\right) \cdot \boldsymbol{\omega}_{t} .
$$

Next we calculate

$$
\partial_{t} \boldsymbol{v}_{t} \cdot \boldsymbol{B}_{\chi}=\boldsymbol{B}_{\chi} \cdot \partial_{t}(\boldsymbol{v}-\sigma \nabla s)=\boldsymbol{B}_{\chi} \cdot\left(\partial_{t} \boldsymbol{v}-\partial_{t} \sigma \nabla s-\sigma \nabla \partial_{t} s\right) .
$$

Taking into account (7.5),

$$
\boldsymbol{B}_{\chi} \cdot \partial_{t} \boldsymbol{v}=\boldsymbol{B}_{\chi} \cdot\left(\boldsymbol{v} \times \boldsymbol{\omega}-\nabla\left(\frac{1}{2} \boldsymbol{v}^{2}+w\right)+T \nabla s\right)+\boldsymbol{B}_{\chi} \cdot \frac{1}{\rho} \boldsymbol{J} \times \boldsymbol{B},
$$

in which the current density is given by

$$
J=\frac{\nabla \times B}{4 \pi} \Rightarrow \nabla \cdot J=0 .
$$

Now,

$$
\boldsymbol{B}_{\chi} \cdot \frac{1}{\rho} \boldsymbol{J} \times \boldsymbol{B}=\frac{1}{\rho} \boldsymbol{J} \cdot \boldsymbol{B} \times \boldsymbol{B}_{\chi}
$$

however,

$$
\boldsymbol{B} \times \boldsymbol{B}_{\chi}=\boldsymbol{B} \times(\nabla \mu \times \nabla \eta)=\nabla \mu(\boldsymbol{B} \cdot \nabla \eta)-\nabla \eta(\boldsymbol{B} \cdot \nabla \mu)=-\rho \nabla \eta .
$$

It thus follows that

$$
\boldsymbol{B}_{\chi} \cdot \frac{1}{\rho} \boldsymbol{J} \times \boldsymbol{B}=-\boldsymbol{J} \cdot \nabla \eta=-\nabla \cdot(\boldsymbol{J} \eta)
$$

in which we used (3.4) and (5.8). Inserting (7.24) into (7.20) will yield

$$
\boldsymbol{B}_{\chi} \cdot \partial_{t} \boldsymbol{v}=\boldsymbol{B}_{\chi} \cdot\left(\boldsymbol{v} \times \boldsymbol{\omega}-\nabla\left(\frac{1}{2} \boldsymbol{v}^{2}+w\right)+T \nabla s\right)-\nabla \cdot(\boldsymbol{J} \eta) .
$$

Inserting (7.25), (7.7) and (7.8) into (7.19) it follows that

$$
\partial_{t} \boldsymbol{v}_{t} \cdot \boldsymbol{B}_{\chi}=\boldsymbol{B}_{\chi} \cdot\left(\boldsymbol{v} \times \boldsymbol{\omega}-\nabla\left(\frac{1}{2} \boldsymbol{v}^{2}+w\right)+(\boldsymbol{v} \cdot \nabla \sigma) \nabla s+\sigma \nabla(\boldsymbol{v} \cdot \nabla s)\right)-\nabla \cdot(\boldsymbol{J} \eta)
$$

hence

$$
\partial_{t} \boldsymbol{v}_{t} \cdot \boldsymbol{B}_{\chi}=\boldsymbol{B}_{\chi} \cdot\left(\boldsymbol{v} \times \boldsymbol{\omega}_{t}+\nabla\left(\sigma(\boldsymbol{v} \cdot \nabla s)-\frac{1}{2} \boldsymbol{v}^{2}-w\right)\right)-\nabla \cdot(\boldsymbol{J} \eta)
$$

Combining (7.18) with (7.27) we arrive at the result

$$
\begin{aligned}
\partial_{t} \boldsymbol{v}_{t} & \cdot \boldsymbol{B}_{\chi}+\boldsymbol{v}_{t} \cdot \partial_{t} \boldsymbol{B}_{\chi} \\
& =\nabla \cdot\left(\left(\boldsymbol{v} \times \boldsymbol{B}_{\chi}\right) \times \boldsymbol{v}_{t}-\boldsymbol{J} \eta\right)+\boldsymbol{B}_{\chi} \cdot \nabla\left(\sigma(\boldsymbol{v} \cdot \nabla s)-\frac{1}{2} \boldsymbol{v}^{2}-w\right) \\
& =\nabla \cdot\left[\left(\boldsymbol{v} \times \boldsymbol{B}_{\chi}\right) \times \boldsymbol{v}_{t}+\boldsymbol{B}_{\chi}\left(\sigma(\boldsymbol{v} \cdot \nabla s)-\frac{1}{2} \boldsymbol{v}^{2}-w\right)-\boldsymbol{J} \eta\right],
\end{aligned}
$$


in which we take into account (7.15). Inserting (7.28) into (7.14) and using Gauss' theorem we obtain a surface integral,

$$
\begin{aligned}
\frac{\mathrm{d} H_{C N B_{\chi}}}{\mathrm{d} t}= & \oint \mathrm{d} \boldsymbol{S} \cdot\left[\left(\boldsymbol{v} \times \boldsymbol{B}_{\chi}\right) \times \boldsymbol{v}_{t}+\boldsymbol{B}_{\chi}\left(\sigma(\boldsymbol{v} \cdot \nabla s)-\frac{1}{2} \boldsymbol{v}^{2}-w\right)-\boldsymbol{J} \eta\right] \\
& -\int \mathrm{d} \boldsymbol{\Sigma} \cdot \boldsymbol{J}[\eta] .
\end{aligned}
$$

The surface integral encapsulates the volume for which the $\chi$ non-barotropic cross-helicity is calculated and an additional surface integral is performed along the cut of $\eta$, in case that $\eta$ is not single valued (see (5.18)). If the surface is taken at infinity the magnetic fields and current densities vanish and thus

$$
\frac{\mathrm{d} H_{C N B \chi}}{\mathrm{d} t}=-\int \mathrm{d} \boldsymbol{\Sigma} \cdot \boldsymbol{J}[\eta] ;
$$

hence for spherical topologies of magnetic field lines or for a current density $\boldsymbol{J}$ parallel to the cut we obtain

$$
\frac{\mathrm{d} H_{C N B \chi}}{\mathrm{d} t}=0,
$$

which means that $H_{C N B \chi}$ is a constant of motion. We notice the complexity of the direct derivation with respect to the elegance and simplicity of the Noether theorem approach. However, obtaining the same result using different methods strengthens our confidence that no mathematical error was accidentally introduced.

\subsection{Direct derivation of the constancy of non-barotropic $\eta$ cross-helicity}

Taking the temporal derivative of the non-barotropic $\eta$ cross-helicity given in $(6.27 a, b)$ we obtain

$$
\frac{\mathrm{d} H_{C N B \eta}}{\mathrm{d} t}=\int \mathrm{d}^{3} x\left[\partial_{t} \boldsymbol{v}_{t} \cdot \boldsymbol{B}_{\eta}+\boldsymbol{v}_{t} \cdot \partial_{t} \boldsymbol{B}_{\eta}\right] .
$$

Let us calculate $\partial_{t} \boldsymbol{B}_{\eta}$ where $\boldsymbol{B}_{\eta}$ is defined in $(6.26 a, b)$ :

$$
\boldsymbol{B}_{\eta}=\nabla \chi \times \nabla \mu
$$

it follows that

$$
\partial_{t} B_{\eta}=\nabla \partial_{t} \chi \times \nabla \mu+\nabla \chi \times \nabla \partial_{t} \mu .
$$

Using (3.3) and (5.7) we obtain

$$
\begin{aligned}
\partial_{t} \boldsymbol{B}_{\eta} & =\nabla(-\boldsymbol{v} \cdot \nabla \chi) \times \nabla \mu+\nabla \chi \times \nabla(-\boldsymbol{v} \cdot \nabla \mu) \\
& =\nabla \times(\nabla \chi(\boldsymbol{v} \cdot \nabla \mu)-\nabla \mu(\boldsymbol{v} \cdot \nabla \chi)) \\
& =\nabla \times(\boldsymbol{v} \times(\nabla \chi \times \nabla \mu))=\nabla \times\left(\boldsymbol{v} \times \boldsymbol{B}_{\eta}\right),
\end{aligned}
$$

in which we used standard vector analysis identities. It thus follows that

$$
\boldsymbol{v}_{t} \cdot \partial_{t} \boldsymbol{B}_{\eta}=\boldsymbol{v}_{t} \cdot \nabla \times\left(\boldsymbol{v} \times \boldsymbol{B}_{\eta}\right)=\nabla \cdot\left(\left(\boldsymbol{v} \times \boldsymbol{B}_{\eta}\right) \times \boldsymbol{v}_{t}\right)+\left(\boldsymbol{v} \times \boldsymbol{B}_{\eta}\right) \cdot \boldsymbol{\omega}_{t} .
$$


Next we calculate

$$
\partial_{t} \boldsymbol{v}_{t} \cdot \boldsymbol{B}_{\eta}=\boldsymbol{B}_{\eta} \cdot \partial_{t}(\boldsymbol{v}-\sigma \nabla s)=\boldsymbol{B}_{\eta} \cdot\left(\partial_{t} \boldsymbol{v}-\partial_{t} \sigma \nabla s-\sigma \nabla \partial_{t} s\right) .
$$

Taking into account (7.5) we have

$$
\boldsymbol{B}_{\eta} \cdot \partial_{t} \boldsymbol{v}=\boldsymbol{B}_{\eta} \cdot\left(\boldsymbol{v} \times \boldsymbol{\omega}-\nabla\left(\frac{1}{2} \boldsymbol{v}^{2}+w\right)+T \nabla s\right)+\boldsymbol{B}_{\eta} \cdot \frac{1}{\rho} \boldsymbol{J} \times \boldsymbol{B} .
$$

Now,

$$
\boldsymbol{B}_{\eta} \cdot \frac{1}{\rho} \boldsymbol{J} \times \boldsymbol{B}=\frac{1}{\rho} \boldsymbol{J} \cdot \boldsymbol{B} \times \boldsymbol{B}_{\eta},
$$

however,

$$
\boldsymbol{B} \times \boldsymbol{B}_{\eta}=\boldsymbol{B} \times(\nabla \chi \times \nabla \mu)=\nabla \chi(\boldsymbol{B} \cdot \nabla \mu)-\nabla \mu(\boldsymbol{B} \cdot \nabla \chi)=\rho \nabla \chi ;
$$

it thus follows that

$$
\boldsymbol{B}_{\eta} \cdot \frac{1}{\rho} \boldsymbol{J} \times \boldsymbol{B}=\boldsymbol{J} \cdot \nabla \chi=\nabla \cdot(\boldsymbol{J} \chi),
$$

in which we used (3.4) and (5.8). Inserting (7.41) into (7.38) will yield

$$
\boldsymbol{B}_{\eta} \cdot \partial_{t} \boldsymbol{v}=\boldsymbol{B}_{\eta} \cdot\left(\boldsymbol{v} \times \boldsymbol{\omega}-\nabla\left(\frac{1}{2} \boldsymbol{v}^{2}+w\right)+T \nabla s\right)+\nabla \cdot(\boldsymbol{J} \chi) .
$$

Inserting (7.42), (7.7) and (7.8) into (7.37) it follows that:

$$
\partial_{t} \boldsymbol{v}_{t} \cdot \boldsymbol{B}_{\eta}=\boldsymbol{B}_{\eta} \cdot\left(\boldsymbol{v} \times \boldsymbol{\omega}-\boldsymbol{\nabla}\left(\frac{1}{2} \boldsymbol{v}^{2}+w\right)+(\boldsymbol{v} \cdot \nabla \sigma) \nabla s+\sigma \nabla(\boldsymbol{v} \cdot \nabla s)\right)+\nabla \cdot(\boldsymbol{J} \chi),
$$

hence

$$
\partial_{t} \boldsymbol{v}_{t} \cdot \boldsymbol{B}_{\eta}=\boldsymbol{B}_{\eta} \cdot\left(\boldsymbol{v} \times \boldsymbol{\omega}_{t}+\nabla\left(\sigma(\boldsymbol{v} \cdot \nabla s)-\frac{1}{2} \boldsymbol{v}^{2}-w\right)\right)+\nabla \cdot(\boldsymbol{J} \chi) .
$$

Combining (7.36) with (7.44) we arrive at the result,

$$
\begin{aligned}
\partial_{t} \boldsymbol{v}_{t} & \cdot \boldsymbol{B}_{\eta}+\boldsymbol{v}_{t} \cdot \partial_{t} \boldsymbol{B}_{\eta} \\
& =\nabla \cdot\left(\left(\boldsymbol{v} \times \boldsymbol{B}_{\eta}\right) \times \boldsymbol{v}_{t}+\boldsymbol{J} \chi\right)+\boldsymbol{B}_{\eta} \cdot \nabla\left(\sigma(\boldsymbol{v} \cdot \nabla s)-\frac{1}{2} \boldsymbol{v}^{2}-w\right) \\
& =\nabla \cdot\left[\left(\boldsymbol{v} \times \boldsymbol{B}_{\eta}\right) \times \boldsymbol{v}_{t}+\boldsymbol{B}_{\eta}\left(\sigma(\boldsymbol{v} \cdot \nabla s)-\frac{1}{2} \boldsymbol{v}^{2}-w\right)+\boldsymbol{J} \chi\right],
\end{aligned}
$$

in which we take into account (7.33). Inserting (7.45) into (7.32) and using Gauss' theorem we obtain a surface integral,

$$
\frac{\mathrm{d} H_{C N B \eta}}{\mathrm{d} t}=\oint \mathrm{d} \boldsymbol{S} \cdot\left[\left(\boldsymbol{v} \times \boldsymbol{B}_{\eta}\right) \times \boldsymbol{v}_{t}+\boldsymbol{B}_{\eta}\left(\sigma(\boldsymbol{v} \cdot \nabla s)-\frac{1}{2} \boldsymbol{v}^{2}-w\right)+\boldsymbol{J} \chi\right] .
$$

The surface integral encapsulates the volume for which the $\eta$ non-barotropic cross-helicity is calculated. If the surface is taken at infinity the magnetic fields and current densities vanish and thus

$$
\frac{\mathrm{d} H_{C N B \eta}}{\mathrm{d} t}=0,
$$

which means that $H_{C N B \eta}$ is a constant of motion. We notice the complexity of the direct derivation with respect to the elegance and simplicity of the Noether theorem approach. However, obtaining the same result using different methods strengthens our confidence that no mathematical error was accidentally introduced. 


\section{Possible application}

\subsection{Bounds and constraints}

In his important review paper 'Physics of magnetically confined plasmas', Boozer (2004) states the following. 'A spiky current profile causes a rapid dissipation of energy relative to magnetic helicity. If the evolution of a magnetic field is rapid, then it must be at constant helicity.' Usually topological conservation laws are used in order to deduce lower bounds on the 'energy' of the flow. Those bounds are only approximate in non-ideal flows but due to their topological nature simulations show that they are approximately conserved even when the 'energy' is not. For example, it is easy to show that the 'energy' is bounded from below by the non-barotropic cross-helicity as follows (see Yahalom 2019a):

$$
\begin{gathered}
\left|H_{C N B}\right|=\left|\int \boldsymbol{B} \cdot \boldsymbol{v}_{t} \mathrm{~d}^{3} x\right| \leq \frac{1}{2} \int\left(\boldsymbol{B}^{2}+\boldsymbol{v}_{t}^{2}\right) \mathrm{d}^{3} x, \\
\left|H_{C N B}\right|=\left|\int \boldsymbol{B} \cdot \boldsymbol{v}_{t} \mathrm{~d}^{3} x\right| \leq \sqrt{\int \boldsymbol{v}_{t}^{2} \mathrm{~d}^{3} x} \sqrt{\int \boldsymbol{B}^{2} \mathrm{~d}^{3} x,}
\end{gathered}
$$

where the second equation is a result of the Cauchy-Schwartz inequality. In this sense a configuration with a highly complicated topology is more stable since its energy is bounded from below. It is a simple thing to show that similar bounds occur also for the $\chi$ and $\eta$ helicities,

$$
\begin{gathered}
\left|H_{C N B \chi}\right|=\left|\int \boldsymbol{B}_{\chi} \cdot \boldsymbol{v}_{t} \mathrm{~d}^{3} x\right| \leq \frac{1}{2} \int\left(\boldsymbol{B}_{\chi}^{2}+\boldsymbol{v}_{t}^{2}\right) \mathrm{d}^{3} x, \\
\left|H_{C N B \chi}\right|=\left|\int \boldsymbol{B}_{\chi} \cdot \boldsymbol{v}_{t} \mathrm{~d}^{3} x\right| \leq \sqrt{\int \boldsymbol{v}_{t}^{2} \mathrm{~d}^{3} x} \sqrt{\int \boldsymbol{B}_{\chi}^{2} \mathrm{~d}^{3} x,} \\
\left|H_{C N B \eta}\right|=\left|\int \boldsymbol{B}_{\eta} \cdot \boldsymbol{v}_{t} \mathrm{~d}^{3} x\right| \leq \frac{1}{2} \int\left(\boldsymbol{B}_{\eta}^{2}+\boldsymbol{v}_{t}^{2}\right) \mathrm{d}^{3} x, \\
\left|H_{C N B \eta}\right|=\left|\int \boldsymbol{B}_{\eta} \cdot \boldsymbol{v}_{t} \mathrm{~d}^{3} x\right| \leq \sqrt{\int \boldsymbol{v}_{t}^{2} \mathrm{~d}^{3} x} \sqrt{\int \boldsymbol{B}_{\eta}^{2} \mathrm{~d}^{3} x .}
\end{gathered}
$$

Hence the kinetic energy is bounded by three different bounds and so it the 'total' energy. The importance of each of those bounds is dependent on the flow.

\subsection{A helical stratified magnetic field}

\subsubsection{The magnetic field and related labels}

Consider a magnetohydrodynamic flow of uniform density $\rho$. Furthermore, assume that the flow contains a helical stratified magnetic field,

$$
\boldsymbol{B}= \begin{cases}2 B_{\perp}\left(1-\frac{R}{a}\right) \hat{\phi}+B_{z 0} \hat{z} & R<a, \\ 0 & R>a,\end{cases}
$$

in which $R, \phi, z$ are the standard cylindrical coordinates, $\hat{R}, \hat{\phi}, \hat{z}$ are the corresponding unit vectors and $B_{z 0}, B_{\perp}$ are constants. The magnetic field is contained in a cylinder of radius 
$a$ and is independent of $z$. Furthermore, we assume that the planes $z=0$ and $z=L$ can be identified such that a topological torus is created. In such a scenario the only field lines that will be closed will satisfy the relation,

$$
\frac{n}{m}=\frac{B_{\perp}}{\pi R B_{z 0}}\left(1-\frac{R}{a}\right) L, \quad n, m \text { integers, }
$$

while lines not satisfying this relation will be surface filling. Nevertheless the magnetic field lines lie on cylindrical surfaces, thus one can calculate the total flux through a circular surface lying on the plane $z$ and bounded by the radius $R$. The magnetic flux in this case will simply be

$$
\Phi=\int \boldsymbol{B} \cdot \mathrm{d} \boldsymbol{S}=\pi R^{2} B_{z 0} .
$$

The $\chi$ function can now be calculated according to (5.9) to yield the value

$$
\chi=\frac{1}{2} B_{z 0} R^{2} .
$$

Solving (5.14) for $\eta$ we obtain the following non-unique solution:

$$
\eta=\phi-\frac{2 B_{\perp}}{B_{z 0}}\left(1-\frac{R}{a}\right) \frac{z}{R}
$$

Finally we solve (5.8) for $\mu$, here we suggest the following simple and non-unique solution:

$$
\mu=\frac{\rho}{B_{z 0}} z
$$

Thus, $\mu$ surfaces are just $z$ planes. Notice that since we have identified the planes $z=0$ and $z=L, \mu$ is non-single-valued. The same can be said of $\eta$ which is doubly non-single-valued in both the $z$ and $\phi$ directions.

\subsubsection{The velocity field}

A stationary velocity field $v$ must satisfy the stationary versions of (2.1) and (2.3),

$$
\begin{gathered}
\nabla \times(\boldsymbol{v} \times \boldsymbol{B})=0, \\
\nabla \cdot(\rho \boldsymbol{v})=0 .
\end{gathered}
$$

Such a velocity field can be constructed using the labels $\mu$ and $\chi$ (see (6.19) of Yahalom \& Lynden-Bell (2008)),

$$
v=k \frac{\nabla \mu \times \nabla \chi}{\rho},
$$

where $k$ is a dimensional constant that we will choose such that $k=v_{0} / a$. Plugging in $\mu$ from (8.12) and $\chi$ from (8.10) we arrive at the simple expression,

$$
v=v_{0} \frac{R}{a} \hat{\phi}
$$


This expression can be shown to solve (2.1) and (2.3) by direct substitution. The stationary version of (2.4) is given by

$$
\rho(\boldsymbol{v} \cdot \nabla) v=-\nabla p+\frac{(\nabla \times B) \times B}{4 \pi} .
$$

This can be solved by the pressure function,

$$
p(R)=\rho\left[\frac{B_{\perp}^{2}}{\pi}\left(3 \frac{R}{a}-\frac{R^{2}}{a^{2}}-\ln \left(\frac{R}{a}\right)-2\right)+\frac{1}{2} v_{0}^{2}\left(\frac{R^{2}}{a^{2}}-1\right)\right], \quad p(a)=0 .
$$

\subsubsection{Cross-helicities}

We now can calculate the cross-helicity using (6.13), in which we assume uniform specific entropy such that $\boldsymbol{v}_{t}=\boldsymbol{v}$. Inserting (8.16) and (8.7) into (6.13) we arrive at the expression

$$
\left|H_{C N B}\right|=\frac{\pi}{3} v_{0} B_{\perp} L a^{2} .
$$

The 'generalized' magnetic fields are calculated using the label scalars as follows:

$$
\boldsymbol{B}_{\chi}=\nabla \mu \times \nabla \eta=\frac{\rho}{B_{z 0}}\left[\frac{2 z B_{\perp}}{B_{z 0} R^{2}} \hat{\phi}-\frac{1}{R} \hat{R}\right], \quad \boldsymbol{B}_{\eta} \equiv \nabla \chi \times \nabla \mu=-\rho R \hat{\phi},
$$

in which we have used $\mu$ given in (8.12), $\chi$ given in (8.10) and $\eta$ given in (8.11). This can be plugged into the generalized cross-helicities defined in $(6.27 a, b)$ to yield the results

$$
\left|H_{C N B \chi}\right|=2 \pi \rho v_{0} \frac{B_{\perp}}{B_{z 0}^{2}} L^{2}, \quad\left|H_{C N B \eta}\right|=\frac{\pi}{2} \rho v_{0} L a^{3} .
$$

Now consider the case that the plasma is initially stationary with the above described density, velocity and magnetic field. Then the plasma is heated such that the density and entropy are not uniform anymore, the pressure thus changes and does not satisfy $(8.18 a, b)$. The plasma is moved from stationary equilibrium and dynamical processes occur (instabilities) in which the velocity and magnetic field are not stationary anymore. Nevertheless, if the evolution satisfies the ideal MHD equation of motion, the values for the cross-helicities given in (8.19) and (8.21a,b) are unmodified and all developing processes and instabilities must satisfy the constraints described in $\S 8.1$.

\section{Conclusion}

We have derived a Noether current from an Eulerian variational principle on non-barotropic MHD, this was shown to lead to the conservation of non-barotropic cross-helicity. The connection of the translation symmetry groups of labels to both the global non-barotropic cross-helicity conservation law and the conservation law of circulations of topological velocity along magnetic field lines was elucidated. The latter were shown to be equivalent to the amount of non-barotropic cross-helicity per unit of magnetic flux (Yahalom 2017b,c, 2019a).

Webb \& Anco (2017) suggested a 19 independent variable variational principle for non-barotropic MHD. They also determined the Lie symmetry responsible for the magnetic helicity conservation law and the magnetic cross-helicity conservation law. 
This symmetry was not a fluid relabeling symmetry, but arose from a complex gauge symmetry of the auxiliary functions they used. In this paper we do not deal with the magnetic helicity conservation law. However, the current paper's simple symmetry leading to the conservation of cross-helicity reflects on the simplicity and elegance of the current variational approach. The reader is invited to compare the complex transformation described in (4.12) of Webb \& Anco (2017) with the simple transformation of the induced label translation given in (6.9). Nevertheless, the approach of Webb \& Anco (2017) allows a derivation of the symmetry behind the magnetic helicity conservation law and it is still an open question if the current simplified formalism is suited for this task.

Much the same can be said of a much older work of Calkin (1963). Calkin suggested an 11 independent variable variational principle for barotropic MHD. He also determined the Lie symmetry responsible for the magnetic helicity conservation law and the magnetic cross-helicity conservation law with respect to his variables. This symmetry was not a fluid relabeling symmetry, but arose from a complex gauge symmetry of the auxiliary functions he used. Again we notice that the simple symmetry of the current approach, leading to the conservation of cross-helicity, reflects on the simplicity and elegance of the current variational principle. The reader is invited to compare the complex transformation described in (88)-(91) of Calkin (1963) with the simple transformation of the induced metage translation given in (6.9).

Furthermore, we have shown that two additional cross-helicity conservation laws exist the $\chi$ and $\eta$ cross-helicities. Those are the easiest ones to obtain as they involve mere label translations. However, it is known from the work of Tur \& Yanovsky (1993) and Sagdeev et al. (1986) on comoving invariants in MHD and in ideal fluids, that many more such constants of topological significance exist. In fact they derived infinite hierarchies of conservation laws associated with fluid relabeling symmetries. We should mention also that for non-magnetic flows Anco \& Webb (2020) also derived hierarchies of conservation laws for the ideal, inviscid, compressible and incompressible fluid equations using Lie dragging techniques.

The new conservation laws lead to new bounds on MHD flows in addition to the bounds of the standard non-barotropic cross-helicity discussed in Yahalom $(2017 c)$ for ideal non-barotropic MHD. The importance of constants of motion for stability analysis is also discussed in Katz, Inagaki \& Yahalom (1993). The significance of those constraints for non-ideal MHD and for plasma physics in general remains to be studied in future works.

It is shown that non-barotropic MHD can be derived from a variational principle of five functions. The formalism is given in a Lagrangian presentation with a geometrical structure.

Possible applications include stability analysis of stationary MHD configurations and its possible utilization for developing efficient numerical schemes for integrating the MHD equations. It may be more efficient to incorporate the developed formalism in the framework of an existing code instead of developing a new code from scratch. Possible existing codes are described in Mignone et al. (2010), Igumenshchev, Narayan \& Abramowicz (2003) and Hoyos, Reisenegger \& Valdivia (2007). Applications of this study may be useful to both linear and nonlinear stability analysis of known barotropic MHD configurations (Bernstein et al. 1958; Almaguer et al. 1988; Katz et al. 1993; Yahalom, Katz \& Inagaki 1994; Vladimirov, Moffatt \& Ilin 1996, 1997, 1999; Yahalom 2011). The theory can also be used to study the evolution of waves with respect to a given MHD configuration, such approach was used by Webb et al. (2005) by introducing a Lagrangian variational principle in which the waves are described by displacements from the background MHD configuration. As for designing efficient numerical schemes 
for integrating the equations of fluid dynamics and MHD, one may follow the approach described in Zhou et al. (2014), Yahalom (2003), Yahalom \& Pinhasi (2003), Yahalom et al. (2005) and Ophir et al. (2012).

Another possible application of the variational method is in deducing new analytic solutions for the MHD equations. Although the equations are notoriously difficult to solve being both partial differential equations and nonlinear, possible solutions can be found in terms of variational variables. An example for this approach is the self-gravitating torus described in Yahalom (2013b), and also in this work in § 8.2.

One can use continuous symmetries which appear in the variational Lagrangian to derive new conservation laws through Noether's theorem. An example for such derivation which still lacks physical interpretation can be found in Yahalom (2013c). It may be that the Lagrangian derived in Yahalom (2010) has a larger symmetry group; and of course one anticipates a different symmetry structure for the non-barotropic case.

Topological invariants have always been informative, and there are such invariants in MHD flows. For example the two helicities have long been useful in research into the problem of hydrogen fusion, and in various astrophysical scenarios. In previous works (Yahalom 1995, 2013a; Yahalom \& Lynden-Bell 2008) connections between helicities with symmetries of the barotropic fluid equations were made. The Noether current here derived may help us to identify and characterize as yet unknown topological invariants in MHD.

\section{Acknowledgements}

This research was supported by the US Department of Energy (DE-AC02-09CH11466).

\section{Declaration of interests}

The authors report no conflict of interest.

\section{REFERENCES}

Almaguer, J. A., Hameiri, E., Herrera, J. \& Holm, D. D. 1988 Lyapunov stability analysis of magnetohydrodynamic plasma equilibria with axisymmetric toroidal flow. Phys. Fluids 31, 1930-1939.

AnCo, S. C. \& WebB, G. M. 2020 Hierarchies of new invariants and conserved integrals in inviscid fluid flow. Phys. Fluids 32, 086104.

Bekenstein, J. D. \& Oron, A. 2000 Conservation of circulation in magnetohydrodynamics. Phys. Rev. E 62, 5594-5602.

Bernstein, I. B., Frieman, E. A., Kruskal, M. D. \& Kulsrud, R. M. 1958 An energy principle for hydromagnetic stability problems. Proc. R. Soc. Lond. A 244, 17-40.

Binney, J. \& Tremaine, S. 1987 Galactic Dynamics. Princeton University Press.

Boozer, A. H. 2004 Physics of magnetically confined plasmas. Rev. Mod. Phys. 76, 1071.

CAlkin, M. G. 1963 An action principle for magnetohydrodynamics. Can. J. Phys. 41, 2241-2251.

Hazeltine, R. D. \& Meiss, J. D. 2003 Plasma Confinement. Dover Books of Physics.

Holm, D. D. \& KupershmidT, B. A. 1983a Poisson brackets and Clebsch representations for magnetohydrodynamics, multifluid plasmas, and elasticity. Physica D 6, 347-363.

Holm, D. D. \& Kupershmidt, B. A. $1983 b$ Noncanonical Hamiltonian formulation of ideal magnetohydrodynamics. Physica D 7, 330-333.

Holm, D. D., Marsden, J. E. \& Ratiu, T. S. 1998 The Euler-Poincaré equations and semidirect products with applications to continuum theories. Adv. Maths 137, 1-81.

Hoyos, J., Reisenegger, A. \& Valdivia, J. A. 2007 Simulation of the magnetic field evolution in neutron stars. VI Reunion Anual Sociedad Chilena de Astronomia (SOCHIAS), vol. 1. p. 20. 
Igumenshchev, I. V., NARAyan, R. \& Abramowicz, M. A. 2003 Three-dimensional magnetohydrodynamic simulations of radiatively inefficient accretion flows. Astrophys. J. 592, $1042-1059$.

KATs, A. V. 2003 Variational principle in canonical variables, Weber transformation, and complete set of the local integrals of motion for dissipation-free magnetohydrodynamics. J. Expl Theor. Phys. Lett. 77, 657-661.

KatZ, J., InAGaki, S. \& Yahalom, A. 1993 Energy principles for self-gravitating barotropic flows: I. General theory. Publ. Astron. Soc. Jpn. 45, 421-430.

LYNDEN-BELL, D. \& KATZ, J. 1981 Isocirculational flows and their Lagrangian and energy principles. Proc. R. Soc. Lond. A 378, 179-205.

Mignone, A., Rossi, P., Bodo, G., Ferrari, A. \& Massaglia, S. 2010 High-resolution 3D relativistic MHD simulations of jets. Mon. Not. R. Astron. Soc. 402, 7-12.

MorRison, P. J. 1982 Poisson brackets for fluids and plasmas. AIP Conf. Proc. 88, 13-46.

Ophir, D., Yahalom, A., Pinhasi, G. A. \& Kopylenko, M. 2012 Fluid simulation: combined Lagrangian and multi-grid approach. Engng Comput. Mech. 165, 3-14.

PAdhye, N. \& Morrison, P. J. $1996 a$ Fluid element relabeling symmetry. Phys. Lett. A 219, 287-292.

PADHYE, N. \& MORRISON, P. J. 1996b Relabeling symmetries in hydrodynamics and magnetohydrodynamics. Plasma Phys. Rep. 22, 869-877.

Sagdeev, R. Z., Moiseev, S. S., Tur, A. V. \& Yanovsky, V. V. 1986 Problems of the theory of strong turbulence and topological solitons. In Nonlinear Phenomena in Plasma Physica and Hydrodynamics (ed. R. Z. Sagdeev), pp. 137-182. Mir.

SAKURAI, T. 1979 A new approach to the force-free field and its application to the magnetic field of solar active regions. Publ. Astron. Soc. Jpn. 31, 209-230.

StUrrock, P. A. 1994 Plasma Physics. Cambridge University Press.

TUR, A. V. \& YANOVSKY, V. V. 1993 Invariants in dissipationless hydrodynamic media. J. Fluid Mech. 248, 67-106.

Vladimirov, V. A. \& Moffatt, H. K. 1995 On general transformations and variational principles for the magnetohydrodynamics of ideal fluids. Part 1. Fundamental principles. J. Fluid Mech. 283, $125-139$.

Vladimirov, V. A., Moffatt, H. K. \& Ilin, K. I. 1996 On general transformations and variational principles for the magnetohydrodynamics of ideal fluids. Part 2. Stability criteria for two dimensional flows. J. Fluid Mech. 329, 187-205.

Vladimirov, V. A., Moffatt, H. K. \& Ilin, K. I. 1997 On general transformations and variational principles for the magnetohydrodynamics of ideal fluids. Part 3. Stability criteria for axisymmetric flows. J. Plasma Phys. 57, 89-120.

Vladimirov, V. A., Moffatt, H. K. \& Ilin, K. I. 1999 On general transformations and variational principles for the magnetohydrodynamics of ideal fluids. Part 4 . Generalized isovorticity principle for three-dimensional flows. J. Fluid Mech. 390, 127-150.

WebB, G. M. \& AnCO, S. C. 2017 On magnetohydrodynamic gauge theory. J. Phys. A 50, 255501.

Webb, G. M., Dasgupta, B., McKenzie, J. F., Hu, Q. \& Zank, G. P. $2014 a$ Local and nonlocal advected invariants and helicities in magnetohydrodynamics and gas dynamics I: Lie dragging approach. J. Phys. A 47, 095501.

Webi, G. M., Dasgupta, B., McKenzie, J. F., Hu, Q. \& Zank, G. P. $2014 b$ Local and nonlocal advected invariants and helicities in magnetohydrodynamics and gas dynamics II: Noether's theorems and Casimirs. J. Phys. A 47, 095502.

WebB, G. M. \& MAce, R. L. 2015 Potential vorticity in magnetohydrodynamics. J. Plasma Phys. 81, 905810115.

Webb, G. M., McKenZIE, J. F. \& ZanK, G. P. 2015 Multi-symplectic magnetohydrodynamics: II. Addendum and erratum. J. Plasma Phys. 81, 905810610.

Webb, G. M., Zank, G. P., Kaghashvili, E. K. \& RatKiewicZ, R. E. 2005 Magnetohydrodynamic waves in non-uniform flows II: stress energy tensors, conservation laws and Lie symmetries. J. Plasma Phys. 71, 811-857.

WoltJer, L. 1958a A theorem on force-free magnetic fields. Proc. Natl Acad. Sci. USA 44, 489-491.

WoltJer, L. $1958 b$ On hydromagnetic equilibrium. Proc. Natl Acad. Sci. USA 44, 833-841. 
Yahalom, A. 1995 Helicity conservation via the Noether theorem. J. Math. Phys. 36, 1324-1327.

YAHALOM, A. 2003 Method and system for numerical simulation of fluid flow. US patent, 6,516,292.

YAHALOM, A. 2010 Barotropic magnetohydrodynamics as a four-function field theory. Europhys. Lett. 89, 34005 .

YAHALOM, A. 2011 Stability in the weak variational principle of barotropic flows and implications for self-gravitating discs. Mon. Not. R. Astron. Soc. 418, 401-426.

Yahalom, A. 2013 a Aharonov-Bohm effects in magnetohydrodynamics. Phys. Lett. A 377, 1898-1904.

YAHALOM, A. $2013 b$ Using fluid variational variables to obtain new analytic solutions of self-gravitating flows with nonzero helicity. Proc. IUTAM 7, 223-232.

YAhaLOM, A. 2013c A new diffeomorphism symmetry group of magnetohydrodynamics. In Lie Theory and its Applications in Physics: IX International Workshop (ed. V. Dobrev), Springer Proceedings in Mathematics and Statistics, vol. 36, pp. 461-468. Springer.

YAHALOM, A. $2016 a$ Simplified variational principles for non-barotropic magnetohydrodynamics. J. Plasma Phys. 82, 905820204.

YAhalom, A. $2016 b$ Non-barotropic magnetohydrodynamics as a five function field theory. Intl J. Geom. Methods M. 13, 1650130.

YAHALOM, A. $2017 a$ A simpler variational principle for stationary non-barotropic ideal magnetohydrodynamics. Chaotic Model. Simul. 1, 19-33.

Yahalom, A. 2017b A conserved local cross helicity for non-barotropic MHD. J. Geophys. Astrophys. Fluid Dyn. 111, 131-137.

YAHALOM, A. 2017c Non-barotropic cross-helicity conservation applications in magnetohydrodynamics and the Aharanov-Bohm effect. Fluid Dyn. Res. 50, 011406.

YAHALOM, A. 2017d Metage symmetry group of non-barotropic magnetohydrodynamics and the conservation of cross helicity. In Quantum Theory and Symmetries with Lie Theory and its Applications in Physics Volume 2 (ed. V. Dobrev), Springer Proceedings in Mathematics and Statistics, vol. 255, pp. 387-402. Springer.

YAHALOM, A. 2019a A new diffeomorphism symmetry group of non-barotropic magnetohydrodynamics. J. Phys.: Conf. Ser. 1194, 012113.

YAHALOM, A. 2019b Topological bounds from label translation symmetry of non-barotropic MHD. J. Phys.: Conf. Ser. 1416, 012041.

YAhalom, A., KATZ, J. \& INAGAKI, S. 1994 Energy principles for self-gravitating barotropic flows: II. The stability of Maclaurin flows. Mon. Not. R. Astron. Soc. 268, 506-516.

YAhalom, A. \& LYNDEN-BELl, D. 2008 Simplified variational principles for barotropic magnetohydrodynamics. J. Fluid Mech. 607, 235-265.

YAhalom, A. \& PINHASI, G. A. 2003 Simulating fluid dynamics using a variational principle. In Proceedings of the 41 st Aerospace Sciences Meeting and Exhibit. AIAA.

Yahalom, A., Pinhasi, G. A. \& Kopylenko, M. 2005 A numerical model based on variational principle for airfoil and wing aerodynamics. In Proceedings of the 43rd AIAA Aerospace Sciences Meeting and Exhibit. AIAA.

Zhou, Y., QIn, H., Burby, J. W. \& BhattacharJee, A. 2014 Variational integration for ideal magnetohydrodynamics with built-in advection equations. Phys. Plasmas 21, 102109. 